\title{
HARMONIC ANALYSIS ON HOMOGENEOUS SPACES
}

\author{
JAE-HYUN YANG
}

\begin{abstract}
This article is an expository paper with some new results. We first survey developments over the past three decades in the theory of harmonic analysis on reductive symmetric spaces. Next we deal with the particular homogeneous space of non-reductive type that is important arithmetically and geometrically. We present some new results on this homogeneous space.
\end{abstract}

\section{Introduction}

Let $G$ be a connected real Lie group and let $H$ be a closed subgroup of $G$. A homogeneous space is understood as a manifold with a transitive action of a Lie group. We see that the coset space $G / H$ is a homogeneous space. We have the natural unitary representation $\pi_{H}$ of $G$ on the Hilbert space $L^{2}(G / H)$ of all square integrable measurable functions on $G / H$ defined by

$$
\left(\pi_{H}(g) f\right)(x)=f\left(g^{-1} x\right),
$$

where $g \in G, x \in G / H$ and $f \in L^{2}(G / H) . \pi_{H}$ is called the (left) regular representation of $G$ for the homogeneous space $G / H$. The fundamental problem in harmonic analysis on $G / H$ is given as follows.

Problem 1. Decompose the regular representation $\pi_{H}$ into irreducible unitary representations of $G$.

We now assume that $\Gamma$ is an arithmetic subgroup of $G$. The right regular representation $\pi_{\Gamma}$ of $G$ on the Hilbert space $L^{2}(\Gamma \backslash G)$ of square integrable measurable functions on $\Gamma \backslash G$ is given by

$$
\left(\pi_{\Gamma}(g) f\right)(z)=f(z g),
$$

where $g \in G, z \in \Gamma \backslash G$ and $f \in L^{2}(\Gamma \backslash G)$. Obviously $\pi_{\Gamma}$ is a unitary representation of $G$. Then we have a basic problem in harmonic analysis on $\Gamma \backslash G$.

Problem 2. Find an explicit formula of the irreducible decomposition of $\pi_{\Gamma}$.

Problem 2 is closely related to the theory of automorphic forms on $G$ for $\Gamma$ and also to the geometry of the associated arithmetic variety. In order to answer the above problems, we need to classify irreducible unitary representations of $G$. Let $\widehat{G}$ be the unitary dual of $G$, that is, the set of all the equivalence classes of irreducible unitary representations of $G$. We denote by $\operatorname{Disc}(G / H)$ (resp. $\operatorname{Disc}(\Gamma \backslash G)$ ) the set of discrete series representations of $G$ for $G / H$ (resp. $\Gamma \backslash G$ ). The first step for solving the above problems might be the determination of the subsets $\operatorname{Disc}(G / H)$ and $\operatorname{Disc}(\Gamma \backslash G)$ of $\widehat{G}$. For instance, it may be meaningful to find a 
criterion under which $\operatorname{Disc}(G / H)$ (resp. $\operatorname{Disc}(\Gamma \backslash G))$ is non-empty. In fact, the study of the unitary dual $\widehat{G}$ has served as a basic tool for the solution of the above problems. We can say that Problem 1 is essentially equivalent to finding an explicit direct integral decomposition

$$
\pi_{H} \cong \int_{\widehat{G}} m_{\pi} \pi d \mu(\pi)
$$

of $\pi_{H}$ into irreducible unitary representations of $G$, where $d \mu$ is a suitable measure (Plancherel measure) on $\widehat{G}$. If $G$ is a connected reductive real Lie group, it can be regarded as a symmetric space $(G \times G) / G$ for the left times right action of $G \times G$. As is well known, in this case (called the group case) an explicit decomposition of the form (1.3) was determined by Harish-Chandra $[26,27,28]$. The components of $L^{2}(G)$ fall in a finite number of series, each of which corresponds to a particular cuspidal parabolic subgroup $P$ and is a direct integral of $\pi_{P, \xi, \lambda} \otimes \pi_{P, \xi, \lambda}^{*}$, where $\pi_{P, \xi, \lambda}$ belongs to the principal series induced from $P=M A N, \xi$ is the discrete series representation of $M$ and $\lambda$ is a continuous parameter ranging in the imaginary linear dual $i \mathfrak{a}$ of the Lie algebra $\mathfrak{a}$ of $A$. The unitary dual $\widehat{G}$ has been investigated by Harish-Chandra, Gelfand school and et al. So far the classification of $\widehat{G}$ has not been solved completely except for some special groups. Recently van den Ban and Schlichkrull $[12,13]$ obtained an explicit Plancherel formula for a reductive symmetric space, which will be explained in Section 4 in some detail. On the other hand, P. Delorme [21] gave the Plancherel formula for a reductive symmetric space in the method different from that of van den Ban and Schlichtkrull. In his paper [19,20], the Maass-Selberg relations for the Eisenstein integrals are established using the truncation method which was inspired by J. Arthur [2]. We refer to [22] for a nice survey on his works.

The last half part of this paper will deal with the example of the special homogeneous space of non-reductive type that is meaningful arithmetically and geometrically. I will explain this roughly.

We consider the Jacobi group $G^{J}=S p(n, \mathbb{R}) \ltimes H_{\mathbb{R}}^{(n, m)}$ which is the semidirect product of the symplectic group $S p(n, \mathbb{R})$ of degree $n$ and the Heisenberg group $H_{\mathbb{R}}^{(n, m)}$ (see (5.14)). $G^{J}$ is not a reductive Lie group and its associated homogeneous space $\mathbb{H}_{n} \times \mathbb{C}^{(m, n)}$ is not a homogeneous space of reductive type, where

$$
\mathbb{H}_{n}=\left\{\Omega \in \mathbb{C}^{(n, n)} \mid \Omega={ }^{t} \Omega, \operatorname{Im} \Omega>0\right\}
$$

is the Siegel upper half plane of degree $n$ and $\mathbb{C}^{(m, n)}$ is the space consisting of $m \times n$ complex matrices. In fact, $G^{J}$ acts on $\mathbb{H}_{n} \times \mathbb{C}^{(m, n)}$ transitively

$$
\left(\left(\begin{array}{ll}
A & B \\
C & D
\end{array}\right),(\lambda, \mu ; \kappa)\right) \cdot(\Omega, Z)=\left((A \Omega+B)(C \Omega+D)^{-1},(Z+\lambda \Omega+\mu)(C \Omega+D)^{-1}\right),
$$

where $M=\left(\begin{array}{ll}A & B \\ C & D\end{array}\right) \in S p(n, \mathbb{R}),(\lambda, \mu ; \kappa) \in H_{\mathbb{R}}^{(n, m)}$ and $(\Omega, Z) \in \mathbb{H}_{n} \times \mathbb{C}^{(m, n)}$. We discuss harmonic analysis on $\mathbb{H}_{n} \times \mathbb{C}^{(m, n)}$ which generalizes the theory of harmonic analysis on $\mathbb{H}_{n}$. We present some new results for the above homogeneous space $\mathbb{H}_{n} \times \mathbb{C}^{(m, n)}$.

This article is organized as follows. In Section 2, we review the harmonic analysis on $\mathbb{R}^{n}$ and compact homogeneous spaces. In Section 3, we survey some results in harmonic analysis on semisimple symmetric spaces. In Section 4, we review harmonic analysis on reductive 
symmetric spaces. We briefly describe some recent results of the Plancherel formula for a reductive symmetric space obtained by van den Ban and Schlichkrull [12, 13, 14, 15]. In Section 5, we discuss the homogeneous space $\mathbb{H}_{n} \times \mathbb{C}^{(m, n)}$ and present some new results. We also give some open problems to be investigated in the future.

Notations: We denote by $\mathbb{R}$ and $\mathbb{C}$ the field of real numbers, and the field of complex numbers respectively. We denote by $\mathbb{Z}$ and $\mathbb{Z}^{+}$be the set of all integers and the set of all positive integers respectively. The symbol ":=" means that the expression on the right is the definition of that on the left. We denote by $C(X)\left(\right.$ resp. $\left.C^{\infty}(X)\right)$ the space of continuous (resp. smooth) functions on a manifold $X$. We denote by $C_{c}^{\infty}(X)$ the space of all smooth functions on $X$ with compact support. For two positive integers $k$ and $l, F^{(k, l)}$ denotes the set of all $k \times l$ matrices with entries in a commutative $\operatorname{ring} F$. For a square matrix $A \in F^{(k, k)}$ of degree $k, \operatorname{tr}(A)$ denotes the trace of $A$. For an $m \times n$ complex matrix $\Omega, \operatorname{Re} \Omega$ (resp. $\operatorname{Im} \Omega$ ) denotes the real (resp. imaginary) part of $\Omega$. For any $M \in F^{(k, l)},{ }^{t} M$ denotes the transpose matrix of $M$. For a matrix $A \in F^{(k, k)}$ and $B \in F^{(k, l)}$, we write $A[B]={ }^{t} B A B$. $I_{n}$ denotes the identity matrix of degree $n$. For a square matrix $A$ of degree $n$, the inequality $A>0$ means that $A={ }^{t} \bar{A}$ is a positive definite hermitian matrix, i.e., ${ }^{t} \bar{x} A x>0$ for any nonzero $x \in \mathbb{C}^{(n, 1)}$. Obviously $A>0$ means in the case of a real $A$ that $A={ }^{t} A$ is a positive definite symmetric matrix, i.e., ${ }^{t} x A x>0$ for any nonzero $x \in \mathbb{R}^{(n, 1)}$. For two $n \times n$ square matrices $A$ and $B$, the inequality $A>B$ means that $A-B>0$, i.e., $A-B$ is a positive definite hermitian matrix. In the real case, the inequality $A>B$ means that $A-B$ is a positive definite symmetric matrix

\section{Harmonic Analysis on $\mathbb{R}^{n}$ and Compact Homogeneous Spaces}

In this section, we review the harmonic analysis on the Euclidean space $\mathbb{R}^{n}$ and some examples of compact homogeneous spaces.

\subsection{Harmonic Analysis on $\mathbb{R}^{n}$}

Let $C_{c}^{\infty}\left(\mathbb{R}^{n}\right)$ be the space of smooth functions on $\mathbb{R}^{n}$ with compact support. Let $($, be the standard inner product on $\mathbb{C}^{n}$ and let $|x|$ be the norm of $x=\left(x_{i}\right) \in \mathbb{C}^{n}$ defined by $|x|^{2}=(x, x)$. The restriction of $($,$) to \mathbb{R}^{n}$ is the standard inner product on $\mathbb{R}^{n}$ and is also denoted by the same notation $($,$) . We denote by \mathrm{PW}\left(\mathbb{R}^{n}\right)$ the space of rapidly decreasing entire functions of exponential type. More precisely, $\varphi$ is an element of $\mathrm{PW}\left(\mathbb{R}^{n}\right)$ if and only if it extends to an entire function on $\mathbb{C}^{n}$ for which there exists a positive number $R>0$ such that the following condition holds for all $N \in \mathbb{Z}^{+}$:

$$
\sup _{\lambda \in \mathbb{C}^{n}}(1+|\lambda|)^{N} e^{-R|\operatorname{Im} \lambda|}|\varphi|<\infty .
$$

If $f$ is an element of $C_{c}^{\infty}\left(\mathbb{R}^{n}\right)$, the Fourier tranform of $f$ is defined by

$$
\widehat{f}(y)=\left(\frac{1}{2 \pi}\right)^{\frac{n}{2}} \int_{\mathbb{R}^{n}} f(x) e^{-i(x, y)} d x .
$$

Then we have the well-known classical results:

$\star$ Inversion Formula : If $f \in C_{c}^{\infty}\left(\mathbb{R}^{n}\right)$, then

$$
f(x)=\left(\frac{1}{2 \pi}\right)^{\frac{n}{2}} \int_{\mathbb{R}^{n}} \widehat{f}(y) e^{i(y, x)} d y .
$$


$\star$ Plancherel Theorem : $f \mapsto \widehat{f}$ extends to an isometry of the Hilbert space $L^{2}\left(\mathbb{R}^{n}\right)$ onto $L^{2}\left(\mathbb{R}^{n}\right)$.

$\star$ Paley-Wiener Theorem : $f \mapsto \widehat{f}$ is a bijection of $C_{c}^{\infty}\left(\mathbb{R}^{n}\right)$ onto the Paley-Wiener space $\mathrm{PW}\left(\mathbb{R}^{n}\right)$.

The above classical Plancherel theorem can be expressed using the unitary representations of the noncompact abelian group $\mathbb{R}^{n}$. First we view $\mathbb{R}^{n}$ as a homogeneous space of $\mathbb{R}^{n}$ acting on itself by translation. The invariant differential operators on $\mathbb{R}^{n}$ are just the differential operators with constant coefficients, and the joint eigenfunctions are the constant multiples of the exponential functions. For brevity, we set $G=\mathbb{R}^{n}$. Then the unitary dual of $G$ is isomorphic to $\mathbb{R}^{n}$. For $\lambda=\left(\lambda_{1}, \cdots, \lambda_{n}\right) \in \mathbb{R}^{n}$, we write

$$
f_{\lambda}(x):=e^{i(\lambda, x)}, \quad x \in \mathbb{R}^{n} \quad \text { and } \quad V_{\lambda}=\mathbb{C} \cdot f_{\lambda} .
$$

A parameter $\lambda=\left(\lambda_{i}\right) \in \mathbb{R}^{n}$ corresponds to the one-dimensional representation $\pi_{\lambda}$ of $G$ on $V_{\lambda}$ defined by

$$
\pi_{\lambda}(g) f_{\lambda}(x):=f_{\lambda}(x-g), \quad g \in G .
$$

Then we have the following Plancherel formula

$$
L^{2}\left(\mathbb{R}^{n}\right)=\int_{\widehat{G}} V_{\lambda} \frac{d \lambda}{(2 \pi)^{n}}=\int_{\mathbb{R}^{n}} V_{\lambda} \frac{d \lambda}{(2 \pi)^{n}}
$$

where $d \lambda$ is the Lebesque measure on $\mathbb{R}^{n}$. In other words, $L^{2}\left(\mathbb{R}^{n}\right)$ is the direct integral of one-dimensional Hilbert spaces $V_{\lambda}$ over the Lebesque measure on $\mathbb{R}^{n}$. The Fourier transform $\widehat{f}$ of $f$ can be considered as a function on $\widehat{G}$.

We can also consider $\mathbb{R}^{n}$ as the homogeneous space $M(n) / O(n)$, where $M(n)=O(n) \ltimes \mathbb{R}^{n}$ is the motion group of $\mathbb{R}^{n}$ equipped with the following multiplication

$$
\left(k_{1}, a_{1}\right) \cdot\left(k_{2}, a_{2}\right)=\left(k_{1} k_{2}, k_{1} a_{2}+a_{1}\right), \quad k_{1}, k_{2} \in O(n), a_{1}, a_{2} \in \mathbb{R}^{n} .
$$

We observe that $O(n)$ is the stabilizer of the action $M(n)$ at $o=(0, \cdots, 0)$. In this case, the Laplacian

$$
L_{\mathbb{R}^{n}}=\frac{\partial^{2}}{\partial x_{1}^{2}}+\cdots+\frac{\partial^{2}}{\partial x_{n}^{2}}
$$

generates the algebra of all $M(n)$-invariant differential operators on $\mathbb{R}^{n}$. We may view a function on $\mathbb{R}^{n}$ as an $L^{2}\left(S^{n-1}\right)$-valued function on $\mathbb{R}^{+}$by means of the geodesic polar coordinates $x=r \omega\left(r>0, \omega \in S^{n-1}\right)$, where $S^{n-1}$ is the $(n-1)$-dimensional unit sphere in $\mathbb{R}^{n}$. Then we can write the Fourier transform $\widehat{f}$ of a function $f$ on $\mathbb{R}^{n}$ in the form

$$
\widehat{f}(r, \omega)=\int_{\mathbb{R}^{n}} f(y) e^{-i(r \omega, y)} d y .
$$

Let $\mathcal{H}:=L_{B}^{2}\left(\mathbb{R}^{+}, r^{n-1} d r\right)$ be the space of $L^{2}\left(S^{n-1}\right)$-valued functions on $\mathbb{R}^{+}$which are square integrable with respect to the measure $r^{n-1} d r$. Then $\widehat{f} \in \mathcal{H}$. The Fourier transform $f \mapsto \widehat{f}$ maps $L^{2}\left(\mathbb{R}^{n}\right)$ isometrically onto $\mathcal{H}$. The decomposition of the left regular representation $\pi_{L}$ of $M(n)$ on $L^{2}\left(\mathbb{R}^{n}\right)$ can be interpreted as follows. First of all, for each $t \in \mathbb{R}$, we define the representation $\rho_{t}$ of $M(n)$ on $L^{2}\left(S^{n-1}\right)$ by

$$
\left(\rho_{t}(k, a) \varphi\right)(\omega)=e^{i(t a, \omega)} \varphi\left(k^{-1} \omega\right), \quad k \in O(n), a \in \mathbb{R}^{n}, \omega \in S^{n-1} .
$$


We can prove that $\rho_{t}$ is irreducible for $t \neq 0$, and that $\rho_{t} \cong \rho_{-t}$. Next we define a unitary representation $\rho$ of $M(n)$ on $\mathcal{H}$ by

$$
(\rho(g) \phi)(t):=\rho_{-t}(g)(\phi(t)), \quad g \in M(n), \phi \in \mathcal{H}, t \in \mathbb{R}^{+} .
$$

Then $\rho$ is isomorphic to the direct integral of the $\rho_{-t}\left(t \in \mathbb{R}^{+}\right)$. Let $\mathbf{1} \in L^{2}\left(S^{n-1}\right)$ be the distinguished vector given by $\mathbf{1}(\omega)=1\left(\omega \in S^{n-1}\right)$. For brevity, we set $H:=O(n)$. If $f \in C_{c}^{\infty}\left(\mathbb{R}^{n}\right)$, then

$$
\widehat{f}(t, \omega)=\int_{M(n)} f(g H) \rho_{-t}(g) \mathbf{1} d g=\rho_{-t}(g) \mathbf{1} .
$$

Therefore the map $f \mapsto \widehat{f}$ is a $M(n)$-equivariant map from $C_{c}^{\infty}\left(\mathbb{R}^{n}\right)$ into $\mathcal{H}$. Hence the map $f \mapsto \widehat{f}$ extends to an isometry of $L^{2}\left(\mathbb{R}^{n}\right)$ onto $\mathcal{H}$, and we have the Plancherel decomposition of $\pi_{L}$ for the homogeneous space $M(n) / H=\mathbb{R}^{n}$. The inversion formula can be reformulated as follows : for $f \in C_{c}^{\infty}\left(\mathbb{R}^{n}\right)$, we get

$$
f(g H)=c \int_{\mathbb{R}^{+}}<\widehat{f}(t), \rho_{-t}(g) \mathbf{1}>r^{n-1} d r
$$

where $c$ is a constant and $<,>$ is an inner product on $L^{2}\left(S^{n-1}\right)$.

\subsection{Harmonic Analysis on Compact Homogeneous Spaces}

Let $G$ be a compact Lie group. It is known that the unitary dual of $G$ is discrete. For $\tau \in \widehat{G}$, we let $\tau^{*}$ be the contragredient (or dual) representation of $G$. As mentioned before, $G$ may be regarded as a homogeneous space $(G \times G) / G$. Indeed the group $G \times G$ acts on $G$ transitively by

$$
(g, h) \cdot x=g x h^{-1}, \quad g, h, x \in G .
$$

The stabilizer $H$ at the identity element $e$ is given by

$$
H=\{(g, g) \in G \times G \mid g \in G\} .
$$

Thus $G$ is diffeomorphic to $(G \times G) / G$. Peter and Weyl [38] proved that the Hilbert space $L^{2}\left(\mathbb{R}^{n}\right)$ is decomposed discretely into

$$
L^{2}(G) \cong L^{2}((G \times G) / G) \cong \sum_{\tau \in \widehat{G}} \tau \otimes \tau^{*}
$$

and each $\tau \in \widehat{G}$ is finite dimensional. This gives the decomposition of the regular representation of $G \times G$ on $L^{2}(G)$ into irreducible subrepresentations of $G \times G$. The formula (2.2) is understood through the matrix coefficient map $\Phi_{\tau}: V_{\tau} \otimes V_{\tau}^{*} \longrightarrow L^{2}(G)$ defined by

$$
\Phi_{\tau}\left(v \otimes v^{*}\right)(g):=\left(\operatorname{dim} V_{\tau}\right)^{1 / 2}<v, \tau^{*}(g) v^{*}>, \quad g \in G, v \in V_{\tau}, v^{*} \in V_{\tau}^{*},
$$

where $V_{\tau}$ is the representation of $\tau \in \widehat{G}$ and $V_{\tau}^{*}$ is its dual space. It is easily seen that the matrix coefficient map $\Phi_{\tau}(\tau \in \widehat{G})$ is a $G \times G$-homomorphism of $V_{\tau} \otimes V_{\tau}^{*}$ into $L^{2}(G)$ with the left times right action. According to the Schur orthogonality relations, $\Phi_{\tau}$ is an injective map preserving the inner products. We identify $V_{\tau} \otimes V_{\tau}^{*}$ with the image $\Phi_{\tau}\left(V_{\tau} \otimes V_{\tau}^{*}\right)$.

Example 2.1. Let $T=S^{1} \times \cdots \times S^{1}$ be the $n$-dimensional torus, where $S^{1}$ is the unit circle in $\mathbb{C}$. Then the unitary dual $\widehat{T}$ is isomorphic to $\mathbb{Z}^{n}$. For each $\alpha=\left(\alpha_{1}, \cdots, \alpha_{n}\right) \in \mathbb{Z}^{n}$, 
we define $h_{\alpha}: T \longrightarrow \mathbb{C}$ by

$$
h_{\alpha}(t)=e^{i\left(\alpha_{1} t_{1}+\cdots+\alpha_{n} t_{n}\right)}, \quad t=\left(t_{1}, \cdots, t_{n}\right) \in T .
$$

We set $W_{\alpha}:=\mathbb{C} \cdot h_{\alpha}$. A parameter $\alpha=\left(\alpha_{i}\right) \in \mathbb{Z}^{n}$ corresponds to the one-dimensional representation $\tau_{\alpha}$ of $T$ on $W_{\alpha}$ defined by

$$
\tau_{\alpha}(g) h_{\alpha}(t):=h_{\alpha}(t-g), \quad g, t \in T .
$$

If $\psi \in L^{2}(T)$, according to Peter-Weyl Theorem, $\psi$ can be written as the direct sum

$$
\psi(t)=\left(\frac{1}{2 \pi}\right)^{\frac{n}{2}} \sum_{\alpha \in \mathbb{Z}^{n}} \widehat{\psi}(\alpha) h_{\alpha}(t)
$$

where

$$
\widehat{\psi}(\alpha)=\left(\frac{1}{2 \pi}\right)^{\frac{n}{2}} \int_{0}^{2 \pi} \cdots \int_{0}^{2 \pi} \psi(t) e^{-i\left(\alpha_{1} t_{1}+\cdots+\alpha_{n} t_{n}\right)} d t_{1} \cdots d t_{n} .
$$

The formula (2.4) is nothing but the Fourier series expansion of a periodic function of period $2 \pi$.

Example 2.2. Let

$$
G=S U(2)=\left\{\left.\left(\begin{array}{cc}
p & q \\
-\bar{q} & \bar{p}
\end{array}\right) \in \mathbb{C}^{(2,2)}|| p\right|^{2}+|q|^{2}=1\right\}
$$

be the special unitary group of degree 2 . For a nonnegative integer $n \geq 0$, we denote by $V_{n}$ the vector space of complex homogeneous polynomials of degree $n$ in two complex variables $z_{1}$ and $z_{2}$. That is, $V_{n}=\sum_{k=0}^{n} \mathbb{C} \cdot z_{1}^{k} z_{2}^{n-k}$. Let $\Phi_{n}$ be the representation of $G$ on $V_{n}$ defined by

$$
\Phi_{n}(g) P\left(\begin{array}{l}
z_{1} \\
z_{2}
\end{array}\right):=P\left(g^{-1}\left(\begin{array}{l}
z_{1} \\
z_{2}
\end{array}\right)\right), \quad g \in G, P \in V_{n} .
$$

We see that $\Phi_{n}$ is an irreducible representation of $G$ and that the unitary dual of $G$ is given by

We recall that for $F \in L^{2}(G)$,

$$
\widehat{G}=\left\{\Phi_{n} \mid n \in \mathbb{Z}, n \geq 0\right\} .
$$

$$
\Phi_{n}(F)=\int_{G} F(g) \Phi_{n}(g) d g
$$

Let $u_{0}, u_{1}, \cdots, u_{n}$ be an orthonormal basis of $V_{n}$. The Hilbert-Schmidt norm $\left\|\Phi_{n}(F)\right\|_{\mathrm{HS}}$ of $\Phi_{n}(F)$ is defined by

$$
\left\|\Phi_{n}(F)\right\|_{\mathrm{HS}}^{2}:=\sum_{i, j=0}^{n}\left|\left(\Phi_{n}(F) u_{i}, u_{j}\right)\right|^{2} .
$$

We observe that it is well defined, i.e., is independent of the choice of an orthonormal basis of $V_{n}$. According to Peter-Weyl theorem, we get the following Plancherel formula

$$
\int_{G}|F(x)|^{2} d x=\sum_{k=0}^{\infty}(k+1)\left\|\Phi_{k}(F)\right\|_{\mathrm{HS}}^{2}, \quad F \in L^{2}(G) .
$$

Let $\Theta_{n}$ be the character of $\Phi_{n}$. Then if $f \in C^{\infty}(G)$, we get the Fourier inversion formula

$$
f\left(I_{2}\right)=\sum_{n=0}^{\infty}(n+1) \Theta_{n}(f) \text {. }
$$


Example 2.3. Let $H$ be a closed subgroup of a compact Lie group $G$. Then the homogeneous space $G / H$ inherits an invariant measure from the Haar measure on $G$. Since $L^{2}(G / H)$ may be identified with the space of right $H$-invariant functions in $L^{2}(G)$, according to the Peter-Weyl theorem, we have the following decomposition

$$
L^{2}(G / H) \cong \oplus_{\tau \in \widehat{G}} V_{\tau} \otimes\left(V_{\tau}^{*}\right)^{H},
$$

where $\left(V_{\tau}^{*}\right)^{H}$ is the $\tau^{*}(H)$-fixed vectors in $V_{\tau}^{*}$. The decomposition (2.5) is orthogonal and equivariant with respect to the $G$-action ( $G$ acts on the tensor product by its action on the first factors), and hence the formula (2.5) gives the decomposition of the regular representation $\pi_{H}$ for $G / H$. We note that the decomposition (2.5) contains only the representation $\tau \in \widehat{G}$ such that $\left(V_{\tau}^{*}\right)^{H} \neq 0$, or equivalently $V_{\tau}^{H} \neq 0$.

Example 2.4. Let $S^{n}$ be the $n$-dimensional sphere in $\mathbb{R}^{n+1}$. Then $S^{n}$ may be regarded as the homogeneous space $O(n+1) / O(n) \cong S O(n+1) / S O(n)$. If we identify $\mathbb{R}^{n+1}$ with $\mathbb{R} \times S^{n}$ using the geodesic polar coordinates, the Laplacian $L_{n}$ on $S^{n}$ is characterized as

$$
L_{\mathbb{R}^{n+1}}=\frac{\partial^{2}}{\partial r^{2}}+\frac{n}{r} \frac{\partial}{\partial r}+\frac{1}{r^{2}} L_{n}
$$

where $L_{\mathbb{R}^{n+1}}$ is the Laplacian on $\mathbb{R}^{n+1}$ (cf. [29]). If $n=2$, using the spherical polar coordinates

on $\mathbb{R}^{3}$, then

$$
x_{1}=r \cos \psi \sin \theta, \quad x_{2}=r \sin \psi \sin \theta, \quad x_{3}=r \cos \theta
$$

where

$$
L_{\mathbb{R}^{3}}=\frac{\partial^{2}}{\partial r^{2}}+\frac{2}{r} \frac{\partial}{\partial r}+\frac{1}{r^{2}} L_{2}
$$

$$
L_{2}=\frac{\partial^{2}}{\partial \theta^{2}}+\cot \theta \frac{\partial}{\partial \theta}+(\sin \theta)^{-2} \frac{\partial^{2}}{\partial \psi^{2}} .
$$

It is known that $L_{n}$ generates the algebra of $O(n+1)$-invariant differential operators on $S^{n}$ (cf. [29], p. 288). Let $s=\left(s_{1}, \cdots, s_{n+1}\right)$ denote the Cartesian coordinates of points on $S^{n}$. The eigenspaces of the Laplacian $L_{n}$ on $S^{n}$ are of the form

$$
E_{k}=\operatorname{span} \text { of }\left\{f_{a, k}(s)=\left(a_{1} s_{1}+\cdots+a_{n+1} s_{n+1}\right)^{k}, s \in S^{n}\right\},
$$

where $k \in \mathbb{Z}^{+} \cup\{0\}$ and $a=\left(a_{1}, \cdots, a_{n+1}\right)$ is an isotropic vector in $\mathbb{C}^{n+1}$, i.e., $a_{1}^{2}+\cdots+$ $a_{n+1}^{2}=0$. The eigenvalue of a function in $E_{k}$ is given by $-k(n-1+k)$. We observe that $E_{k}$ is invariant under the action of $O(n+1)$. We can show that the eigenspace representation $T_{k}\left(k \in \mathbb{Z}^{+} \cup\{0\}\right)$ of $O(n+1)$ on $E_{k}$ defined by

$$
\left(T_{k}(g) f\right)(s)=f\left(g^{-1} \cdot s\right), \quad g \in O(n+1), s \in S^{n}, f \in E_{k}
$$

is irreducible.

Let $P_{k}$ be the space of homogeneous polynomials of degree $k$ on $\mathbb{R}^{n+1}$ and let $H_{k} \subset P_{k}$ the subspace of harmonic polynomials. We define the bilinear form $<$, > on $P\left(\mathbb{R}^{n+1}\right):=$ $\sum_{k=0}^{\infty} P_{k}$ by

$$
<p, q>:=\left\{p\left(\frac{\partial}{\partial x_{1}}, \cdots, \frac{\partial}{\partial x_{n+1}}\right) q\right\}(0), \quad p, q \in P\left(\mathbb{R}^{n+1}\right) .
$$

It is easily checked that $<,>$ is symmetric, positive definite and that

$$
P_{k}=q P_{k-2}+H_{k}, \quad q(x):=x_{1}^{2}+\cdots+x_{n+1}^{2} .
$$


We can see that the restriction map $\left.p \mapsto p\right|_{S^{n}}$ of $H_{k}$ onto $E_{k}$ is one-to-one. The eigenfunctions of $L_{n}$ on $S^{n}$ are called spherical harmonic. The name is derived from the fact that they coincide with the set of restrictions $\cup_{k>0}\left(\left.H_{k}\right|_{S^{n}}\right)$.

The Hilbert space $L^{2}\left(S^{n}\right)$ is decomposed into

$$
L^{2}\left(S^{n}\right)=\sum_{k=0}^{\infty} E_{k} \quad \text { (orthogonal sum for }<,>\text { ). }
$$

The regular representation $\rho$ of $O(n+1)$ on $S^{n}$ is mutiplicity-free, that is, the decomposition (2.6) is a multiplicity-free decomposition of $\rho$. Let $d(k)$ be the dimension of $E_{k}$. Let $\left\{S_{k, m} \mid 1 \leq m \leq d(k)\right\}$ be an orthonormal basis of $E_{k}$. For $f \in C^{\infty}\left(S^{n}\right)$, the series

$$
\sum_{k, m} a_{k, m} S_{k, m}, \quad a_{k, m}:=<f, S_{k, m}>
$$

converges absolutely and uniformly to $f$. The mapping $f \mapsto\left\{a_{k, m}\right\}$ sends $C^{\infty}\left(S^{n}\right)$ onto the set of all sequences $\left\{a_{k, m} \mid k \in \mathbb{Z}^{+} \cup\{0\}, 1 \leq m \leq d(k)\right\}$ satisfying the condition

$$
\sup _{k, m}\left|a_{k, m}\right| k^{q}<\infty
$$

for each $q \in k \in \mathbb{Z}^{+} \cup\{0\}$. For more detail, we refer to [29].

\section{Semisimple Symmetric Spaces}

Let $G$ be a connected semisimple Lie group. Let $\theta$ be a Cartan involution of $G$, that is, an involution whose fixed subgroup $K:=G^{\theta}=\{g \in G \mid \theta(g)=g\}$ is a maximal compact subgroup of $G$. Let $\sigma$ be an involution of $G$ commuting with $\theta$. Let $H$ be an open subgroup of the group $G^{\sigma}$ of fixed points for $\sigma$. Then the homogeneous space $X=G / H$ is called a semisimple symmetric space.

Let $\mathfrak{g}$ be the Lie algebra of $G$, and let $\mathfrak{k}$ and $\mathfrak{h}$ be the subalgebras of $\mathfrak{g}$ corresponding to $K$ and $H$ respectively. The differential of $\theta$ (resp. $\sigma$ ) is also denoted by the same symbol $\theta$ (resp. $\sigma$ ). Then we have the following decomposition

$$
\mathfrak{g}=\mathfrak{k} \oplus \mathfrak{p}=\mathfrak{h} \oplus \mathfrak{q}
$$

of $\mathfrak{g}$ into the \pm 1 eigenspaces for $\theta$ and $\sigma$ respectively. We note that $\mathfrak{p}$ and $\mathfrak{q}$ are the orthogonal complements of $\mathfrak{k}$ and $\mathfrak{h}$ with respect to the Killing form respectively. Since $\theta$ and $\sigma$ commute with each other, we get the joint decomposition

$$
\mathfrak{g}=\mathfrak{k} \cap \mathfrak{h} \oplus \mathfrak{h} \cap \mathfrak{p} \oplus \mathfrak{k} \cap \mathfrak{q} \oplus \mathfrak{p} \cap \mathfrak{q} .
$$

A Cartan subspace $\mathfrak{b}$ for $G / H$ is defined to a maximal abelian subspace of $\mathfrak{q}$ that consists of semisimple elements. All Cartan subspaces have the same dimension, which is called the rank of $G / H$. We say that a Cartan subspace $\mathfrak{b}$ is fundamental if $\mathfrak{k} \cap \mathfrak{b}$ is maximal abelian in $\mathfrak{k} \cap \mathfrak{q}$, and that it is maximal split if $\mathfrak{b} \cap \mathfrak{p}$ is maximal abelian in $\mathfrak{q} \cap \mathfrak{p}$. There exist, up to conjugation by $K \cap H$, a unique fundamental Cartan subspace and a unique maximal split Cartan subspace for $G / H$. If the fundamental Cartan subspace for $G / H$ is contained in $\mathfrak{k}$, it is called a compact Cartan subspace. The dimension of the $\mathfrak{p}$-part of a maximal split Cartan subspace for $G / H$ is called the split rank of $G / H$. 
Here are examples of semisimple symmetric spaces:

$$
\begin{aligned}
S O(n+1) / S O(n)= & \text { the } n \text {-dimensional sphere, } \\
S L(n, \mathbb{R}) / S O(m, n-m)= & \text { the space of quadratic forms of signature }(m, n-m) \text { in } \mathbb{R}^{n}, \\
& \quad \text { where } 0 \leq m \leq n, \\
S p(n, \mathbb{R}) / U(n)= & \text { the Siegel upper half plane of degree } n, \\
S O_{0}(p, q+1) / S O_{0}(p, q)= & \text { the hyperboloid } \mathbb{H}^{p, q} \text { in } \mathbb{R}^{p+q+1} .
\end{aligned}
$$

Here

$$
\mathbb{H}^{p, q}=\left\{x=\left(x_{k}\right) \in \mathbb{R}^{p+q+1} \mid \sum_{i=1}^{p} x_{i}^{2}-\sum_{j=p+1}^{p+q+1} x_{j}^{2}=-1\right\}
$$

and if $q=0$, we should take a connected component of $\mathbb{H}^{p, 0}$.

Another important example is the group case mentioned before. Let $G$ be a semisimple Lie group. Then $G_{*}:=G \times G$ acts on $G$ transitively via (2.1). Since the stabilizer $d\left(G_{*}\right)$ at the identity $e$ is given by

$$
d\left(G_{*}\right)=\left\{(g, g) \in G_{*} \mid g \in G\right\},
$$

$G=G_{*} / d\left(G_{*}\right)$. We let $\sigma$ be the involution of $G$ defined by

$$
\sigma(g, h)=(h, g), \quad g, h \in G .
$$

Then the fixed group $H=G^{\sigma}$ for $\sigma$ is equal to $d\left(G_{*}\right)$. Hence $G=G_{*} / H$ is a semisimple symmetric space.

Let $\pi_{H}$ be the regular representation of $G$ for a semisimple symmetric space $G / H$ defined by (1.1). Let $L_{d}^{2}(G / H)$ be the closed linear span of the irreducible subrepresentations of $\left(\pi_{H}, L^{2}(G / H)\right)$. An irreducible representation $\pi$ is said to be a discrete series representation for $G / H$ if it is realized in a closed subspace $L_{d}^{2}(G / H)$ of $L^{2}(G / H)$. We denote by $\operatorname{Disc}(G / H)$ the set of all discrete series representations for $G / H$.

Theorem 3.1 (Flensted-Jensen [24], Oshima-Matsuki $[35,36,37])$. Let $G / H$ be a semisimple symmetric space. Then $L_{d}^{2}(G / H)$ is nonzero (equivalently $\operatorname{Disc}(G / H)$ is nonempty) if and only if $\operatorname{rank}(G / H)=\operatorname{rank}(K / K \cap H)$.

This theorem generalizes the work of Harish-Chandra.

Corollary 3.2 (Harish-Chandra [25]). Let $G$ be a connected semisimple Lie group. Then the following are equivalent :

(1) $L_{d}^{2}(G) \neq 0$, equivalently $\operatorname{Disc}(G) \neq \emptyset$.

(2) $\operatorname{rank}(G)=\operatorname{rank}(K)$, where $K$ is a maximal compact subgroup of $G$.

(3) $G$ has a compact Cartan subgroup.

Examples 3.3. (a) If $G=S p(n, \mathbb{R})(n \geq 1), S U(p, q)$ with $p q$ even, then $\operatorname{Disc}(G) \neq \emptyset$.

(b) If $G=S O(p, q)$ with $p q$ even, then $L_{d}^{2}(G) \neq 0$.

(c) If $G=S L(n, \mathbb{R})(n>2), S O(p, q)$ with $p q$ odd, and if $G$ is any complex Lie group, then $\operatorname{Disc}(G)=\emptyset$. 
Remark 3.4. H. Schlichtkrull [39] and T. Kobayashi [31] generalized the above theorem by constructing discrete series representations for vector bundles over symmetric spaces.

The Plancherel formula for $G / H$ is understood as a formula decomposing the regular representation $\pi_{H}$ into irreducible unitary representations of $G$. In the case of semisimple symmetric spaces, the Plancherel formula for $G / H$ was obtained by Peter-Weyl (the compact group case), Gelfand-Naimark (classical complex semisimple Lie groups), Harish-Chandra (real semisimple Lie groups) and Helgason (Riemannian symmetric space; $\operatorname{Disc}(G / H)=\emptyset$ ). We may say that the determination of $\operatorname{Disc}(G / H)$ plays an important role in obtaining the Plancherel formula.

Example 3.5. We shall describe briefly the harmonic analysis on the unit disk $\mathbb{D}$. The unit disk

$$
\mathbb{D}=\{w \in \mathbb{C}|| w \mid<1\}
$$

is realized as the homogeneous space $S U(1,1) / K$, where

and

$$
S U(1,1)=\left\{\left.\left(\begin{array}{cc}
p & q \\
\bar{q} & \bar{p}
\end{array}\right)|| p\right|^{2}-|q|^{2}=1, p, q \in \mathbb{C}\right\}
$$

$$
K=\left\{\left(\begin{array}{cc}
p & 0 \\
0 & \bar{p}
\end{array}\right)|| p \mid=1, p \in \mathbb{C}\right\} .
$$

We note that $S U(1,1)$ acts on $\mathbb{D}$ transitively by

$$
\left(\begin{array}{cc}
p & q \\
\bar{q} & \bar{p}
\end{array}\right) \cdot w=(p w+q)(\bar{q} w+\bar{p})^{-1}, \quad w \in \mathbb{D}
$$

and that $K$ is the stabilizer of the above action at the origin $o$. Thus $\mathbb{D}$ is a Riemannian symmetric space of noncompact type. The metric

$$
d s^{2}=\frac{d w d \bar{w}}{\left(1-|w|^{2}\right)^{2}}=\frac{d u^{2}+d v^{2}}{\left(1-u^{2}-v^{2}\right)^{2}}, \quad w=u+i v \in \mathbb{D}
$$

is a Riemannian metric invariant under the action $(3.3)$ of $S U(1,1)$ and its Laplacian $\Delta$ is given by

$$
\Delta=4\left(1-|w|^{2}\right)^{2} \frac{\partial^{2}}{\partial w \partial \bar{w}}=\left(1-u^{2}-v^{2}\right)^{2}\left(\frac{\partial^{2}}{\partial u^{2}}+\frac{\partial^{2}}{\partial v^{2}}\right) .
$$

The differential form

$$
d V=\left(1-u^{2}-v^{2}\right)^{-2} d u \wedge d v
$$

is a $S U(1,1)$-invariant volume element on $\mathbb{D}$. Let $B:=\{w \in \mathbb{C}|| w \mid=1\}$ be the boundary of $\mathbb{D}$. Then the geodesics in $\mathbb{D}$ are the circular arcs perpendicular to $B$. The distance between two points $w_{1}$ and $w_{2}$ in $\mathbb{D}$ with the respect to the metric (3.4) is given by

$$
d\left(w_{1}, w_{2}\right)=\frac{1}{2} \log \frac{\left|1-\bar{w}_{1} w_{2}\right|+\left|w_{2}-w_{1}\right|}{\left|1-\bar{w}_{1} w_{2}\right|-\left|w_{2}-w_{1}\right|} .
$$

A circle in $\mathbb{D}$ tangential to $B$ is called a horocycle. We see that a horocycle $\xi$ in $\mathbb{D}$ is perpendicular to all geodesics in $\mathbb{D}$ tending to the point of contact of $\xi$. For a point $w$ on a horocycle $\xi$, with the point $b$ of contact, we define

$$
<w, b>:=\text { distance from } o \text { to } \xi,
$$


where the negative sign is taken if $o$ lies inside $\xi$. According to (3.6) and the cosine relation on the triangle, we see that

$$
e^{2<w, b>}=\frac{1-|w|^{2}}{|w-b|^{2}}, \quad w \in \xi
$$

Let $\mathcal{S}(B)$ be the space of analytic functions on $B$ and let $\mathcal{S}^{\prime}(B)$ its dual space. Since the elements of $\mathcal{S}^{\prime}(B)$ generalize measures, it is convenient to write

$$
T(f)=\int_{B} f(b) d T(b), \quad f \in \mathcal{S}(B), T \in \mathcal{S}^{\prime}(B) .
$$

For $\lambda \in \mathbb{C}$, we let

$$
E_{\lambda}(\mathbb{D}):=\left\{f \in C^{\infty}(\mathbb{D}) \mid \Delta f=-\left(\lambda^{2}+1\right) f\right\}
$$

be the eigenspace of $\Delta$ with the topology induced from that of $C^{\infty}(\mathbb{D})$. It is known (cf. [29]) that the eigenspace representation $T_{\lambda}$ of $S U(1,1)$ on $E_{\lambda}(\mathbb{D})$ is irreducible if and only if $i \lambda+1$ is not an even integer. We can show that the functions

$$
f_{\lambda, T}(w):=\int_{B} e^{(i \lambda+1)<w, b>} d T(b), \quad \lambda \in \mathbb{C}, T \in \mathcal{S}^{\prime}(B)
$$

exhaust all the eigenfunctions of the Lapalcian $\Delta$, and moreover that if $i \lambda \neq-1,-3,-5, \cdots$, then the mapping $T \mapsto f_{T, \lambda}$ from $\mathcal{S}^{\prime}(B)$ to $E_{\lambda}(\mathbb{D})$ is bijective.

A spherical function on $\mathbb{D}$ is by definition a radial eigenfunction of $\Delta$. If $w \in \mathbb{D}$ and $r=d(0, w)$, we see from (3.7) that

$$
w=|w| e^{i \theta}=\tanh r e^{i \theta} .
$$

In the geodesic polar coordinates $(r, \theta), \Delta$ is given by

$$
\Delta=\frac{\partial^{2}}{\partial r^{2}}+2 \operatorname{coth} 2 r \frac{\partial}{\partial r}+4 \sinh ^{-2}(2 r) \frac{\partial^{2}}{\partial \theta^{2}} .
$$

Thus a spherical function $\phi$ satisfies the differential equation

$$
\frac{\partial^{2} \phi}{\partial r^{2}}+2 \operatorname{coth} 2 r \frac{\partial \phi}{\partial r}=-\left(\lambda^{2}+1\right) \phi .
$$

The following function $\phi_{\lambda}$ defined by

$$
\phi_{\lambda}(w):=\int_{B} e^{(i \lambda+1)<w, b>} d b, \quad \lambda \in \mathbb{C}
$$

satisfies the equation (3.10). By expanding a power series in sinh $2 r$, we see that all smooth solutions of (3.10) are the constant multiples of $\phi_{\lambda}(w)$.

We put

$$
a_{t}:=\left(\begin{array}{ll}
\cosh t & \sinh t \\
\sinh t & \cosh t
\end{array}\right) \in S U(1,1), \quad t \in \mathbb{R}
$$

We can show that if $\operatorname{Re}(i \lambda)>0$, the limit

$$
\mathbf{c}(\lambda):=\lim _{t \longrightarrow \infty} e^{(-i \lambda+1) t} \phi_{\lambda}\left(a_{t} \cdot o\right)
$$

exists and

$$
\mathbf{c}(\lambda)=\pi^{-1 / 2} \frac{\Gamma\left(\frac{1}{2} i \lambda\right)}{\Gamma\left(\frac{1}{2}(i \lambda+1)\right)}
$$


where $\Gamma(x)$ is the Gamma function. The formula (3.12) defines a meromorphic function in $\mathbb{C}$, which is called the Harish-Chandra's c-function.

Definition. If $f$ is a radial function on $\mathbb{D}$, its spherical transform $\tilde{f}$ is defined by

$$
\tilde{f}(\lambda):=\int_{\mathbb{D}} f(w) \phi_{-\lambda}(w) d V
$$

whenever this integral converges. Here $d V$ denotes a $S U(1,1)$-invariant volume element given by (3.6).

It can be proved (cf. [29], p. 40) that if $f$ is a radial function in $C_{c}^{\infty}(\mathbb{D})$,

$$
f(w)=\frac{1}{2 \pi^{2}} \int_{\mathbb{R}} \tilde{f}(\lambda) \phi_{\lambda}(w)|\mathbf{c}(\lambda)|^{-2} d \lambda
$$

and

$$
\int_{\mathbb{D}}|f(w)|^{2} d V=\frac{1}{2 \pi^{2}} \int_{\mathbb{R}}|\tilde{f}(\lambda)|^{2}|\mathbf{c}(\lambda)|^{-2} d \lambda .
$$

Definition. If $f$ is a complex-valued function on $\mathbb{D}$, its Fourier transform $\tilde{f}$ is defined by

$$
\tilde{f}(\lambda, b):=\int_{\mathbb{D}} f(w) e^{(-i \lambda+1)<z, b>} d V
$$

for all $\lambda \in \mathbb{C}, b \in B$ for which this integral exists.

Then we have the following results:

$\star$ Inversion Formula : If $f \in C_{c}^{\infty}(\mathbb{D})$, then

$$
f(w)=\frac{1}{4 \pi} \int_{\mathbb{R}} \int_{B} \tilde{f}(\lambda, b) e^{(i \lambda+1)<w, b>} \lambda \tanh \left(\frac{\pi \lambda}{2}\right) d \lambda d b,
$$

where $d \lambda$ is the Lebesque measure on $\mathbb{R}$ and $d b$ is the circular measure on $B$ normalized by $\int_{B} d b=1$.

$\star$ Paley-Wiener Theorem : $f \mapsto \tilde{f}$ is a bijection of $C_{c}^{\infty}(\mathbb{D})$ onto the Paley-Wiener space $\mathrm{PW}(\mathbb{D})$, where $\mathrm{PW}(\mathbb{D})$ is the space of smooth functions $\psi: \mathbb{C} \times B \longrightarrow \mathbb{C}$ which is holomorphic in $\mathbb{C}$ such that if for each $N \in \mathbb{Z}^{+} \cup\{0\}$ and $R>0$,

$$
\sup _{\lambda \in \mathbb{C}, b \in B} e^{-R|\operatorname{Im} \lambda|}(1+|\lambda|)^{N}|\psi(\lambda, b)|<\infty
$$

and

$$
\int_{B} e^{(i \lambda+1)<w, b>} \psi(\lambda, b) d b=\int_{B} e^{(-i \lambda+1)<w, b>} \psi(-\lambda, b) d b
$$

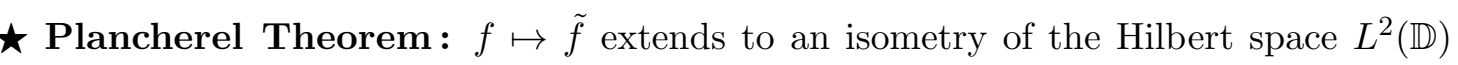
onto

$$
L^{2}\left(\mathbb{R}^{+} \times B,(2 \pi)^{-1} \lambda \tanh \left(\frac{\pi \lambda}{2}\right) d \lambda d b\right)
$$

where $\mathbb{R}^{+}$denotes the set of all nonnegative real numbers.

We refer to [29] for the proof of the above results. Since $\mathbb{D}=S U(1,1) / K$ is a Riemannian symmetric space of noncompact type, the decomposition of $L^{2}(\mathbb{D})$ contains no discrete series representation. 
Example 3.6. If $X=S O_{0}(p, q) / S O_{0}(p-1, q), S U(p, q) /(S(U(1) \times U(p-1, q))$, or $S p(p, q) /(S p(1) \times S p(p-1, q))(p>1, q>0)$, then $X$ is a semisimple symmetric space. The decomposition of $L^{2}(X)$ contains both a discrete part (discrete series) and a continuous part (the principal series). In particular, it is known that the multiplicities are one, except when $X=S O_{0}(p, 1) / S O_{0}(p-1,1)$, where the representations of the principal series have multiplicity 2 (cf. [23]).

\section{Homogeneous Spaces of Reductive Type}

In this section, for simplicity we assume that $G$ is a real reductive linear group. By definition, $G$ is a connected closed subgroup of the general linear group $G L(N, \mathbb{R})$ which are stable under the standard Cartan involution $\theta_{0}: G \longrightarrow G$ defined by $\theta_{0}(g)={ }^{t} g^{-1}(g \in G)$. Next we assume that $H$ is a closed subgroup of $G$ and that both $G$ and $H$ have at most finitely many connected components. The coset space $G / H$ is said to be a homogeneous space of reductive type if $G$ and $H$ are realized as closed subgroups of $G L(N, \mathbb{R})$ such that $G L(N, \mathbb{R}) \supset G \supset H$ are stable under the standard Cartan involution $\theta_{0}$ (cf. [32]).

We give some examples of reductive homogeneous spaces of reductive type.

$$
\begin{aligned}
G L(n, \mathbb{R}) / O(n) & =\text { the cone of } n \times n \text { positive real symmetric spaces, } \\
G L(2 n, \mathbb{R}) / G L(n, \mathbb{C}) & =\text { the space of complex structures on } \mathbb{R}^{2 n}, \\
U(p, q ; \mathbb{F}) / U(p-m, q ; \mathbb{F}) & =\text { the indefinite Stiefel manifold, where } \mathbb{F}=\mathbb{R}, \mathbb{C}, \mathbb{H} .
\end{aligned}
$$

Here $\mathbb{H}$ denotes the quaternionic number field and $U(p, q ; \mathbb{H}) \cong S p(p, q)$.

Let $G$ be a real reductive linear group and let $\theta$ be a Cartan involution of $G$. The differential of $\theta$ is also denoted by the same symbol $\theta$. We have the so-called Cartan decomposition of $\mathfrak{g}=\mathfrak{k}+\mathfrak{p}$ of $\mathfrak{g}$, where $\mathfrak{k}$ and $\mathfrak{p}$ are the \pm 1 eigenspaces of $\theta$ respectively. There exists a $G$-invariant nondegenerate symmetric $\mathbb{R}$-bilinear form $B$ on $\mathfrak{g}$ such that the bilinear form $B_{\theta}: \mathfrak{g} \times \mathfrak{g} \longrightarrow \mathbb{R}$ defined by

$$
B_{\theta}(X, Y):=-B(X, \theta Y), \quad X, Y \in \mathfrak{g}
$$

is symmetric and positive definite. If $G$ is a simple Lie group, $B$ coincides with the Killing form of $\mathfrak{g}$ up to positive multiples. For instance, we realize $G$ as a subgroup of $G L(N, \mathbb{R})$ which is stable under $\theta_{0}$. The differential $\theta_{0}$ of $\theta_{0}$ is given by $\theta_{0}(X)=-{ }^{t} X(X \in \mathfrak{g l}(n, \mathbb{R}))$. We set

$$
\begin{aligned}
K: & =O(n) \cap G, \\
\mathfrak{k}: & =\mathfrak{g} \cap\{N \times N \text { real skew-symmetric matrices }\}, \\
\mathfrak{p}: & =\mathfrak{g} \cap\{N \times N \text { real symmetric matrices }\}, \\
B(X, Y): & =\operatorname{tr}(X Y), \quad X, Y \in \mathfrak{g}, \\
B_{\theta_{0}}(X, Y): & =\operatorname{tr}\left(X^{t} Y\right), \quad X, Y \in \mathfrak{g},
\end{aligned}
$$

where $\operatorname{tr}(X)$ denotes the trace of an $N \times N$ matrix. It is easily seen that $B_{\theta_{0}}$ is positive definite.

We assume that $\sigma$ is an involution of $G$ commuting with a Cartan involution $\theta$. Let $H$ be an open subgroup of the group $G^{\sigma}$ of fixed points for $\sigma$. Namely $G^{\sigma}=\{g \in G \mid \sigma(g)=g\}$. The coset space $G / H$ is said to be a reductive symmetric space. 
We survey the recent progress in harmonic analysis on reductive symmetric spaces. First we describe the recent works of van den Ban and Schlichtkrull (cf. [3]-[15]). Let $\mathfrak{k}$ and $\mathfrak{h}$ be the subalgebras of $\mathfrak{g}$ corresponding to $K$ and $H$ respectively. Then we have the decomposition (3.1) and (3.2). Let $\mathfrak{a}$ be a maximal abelian subalgebra of $\mathfrak{g}$. We set $\mathfrak{a}_{\mathfrak{q}}:=\mathfrak{a} \cap \mathfrak{q}$. Let $P=M_{P} A_{P} N_{P}$ be a Langlands decomposition of $\sigma \theta$-stable parabolic subgroup $P$ of $G$ containing $A_{\mathfrak{q}}:=\exp \mathfrak{a}_{\mathfrak{q}}$. Let $\mathfrak{m}_{P}, \mathfrak{a}_{P}$ and $\mathfrak{n}_{P}$ be the subalgebra of $\mathfrak{g}$ corresponding to the subgroups $M_{P}, A_{P}$ and $N_{P}$ respectively. We denote by $\widehat{M_{P}}$ the unitary dual of $M_{P}$ and let $\mathfrak{a}_{P \mathbb{C}}^{*}:=\operatorname{Hom}_{\mathbb{R}}\left(\mathfrak{a}_{P}, \mathbb{C}\right)$ be the complexified linear dual of $\mathfrak{a}_{P}$. Let $\rho_{P}$ be the element of $\mathfrak{a}_{P}^{*}$ defined by

$$
\rho_{P}(a):=\frac{1}{2} \operatorname{tr}\left(\left.\operatorname{ad}(a)\right|_{\mathfrak{n}_{P}}\right), \quad a \in \mathfrak{a}_{P}
$$

For $\left(\xi, \mathcal{H}_{\xi}\right) \in \widehat{M_{P}}$ and $\lambda \in \mathfrak{a}_{P \mathbb{C}}^{*}$, we define the induced representation

$$
\pi_{P, \xi, \lambda}:=\operatorname{Ind}_{P}^{G}\left(\xi \otimes e^{\lambda} \otimes 1\right)
$$

as follows: The representation space $\mathcal{H}_{P, \xi, \lambda}$ of $\pi_{P, \xi, \lambda}$ is the Hilbert space of measurable functions $f: G \longrightarrow \mathcal{H}_{\xi}$ satisfying the transformation behavior

$$
f(\operatorname{man} x)=a^{\lambda+\rho_{P}} \xi(m) f(x),
$$

where $m \in M_{P}, a \in A_{P}, n \in N_{P}$ and $x \in G$. The inner product on $\mathcal{H}_{P, \xi, \lambda}$ is given by

$$
<f, g>:=\int_{K}<f(k), g(k)>_{\mathcal{H}_{\xi}} d k, \quad f, g \in \mathcal{H}_{P, \xi, \lambda},
$$

where $d k$ denotes the normalized Haar measure on $K . \pi_{P, \xi, \lambda}$ is realized in $\mathcal{H}_{P, \xi, \lambda}$ as

$$
\pi_{P, \xi, \lambda}(g) f(x)=f(x g), \quad g, x \in G, f \in \mathcal{H}_{P, \xi, \lambda} .
$$

Let $W_{P}$ denote the centralizer of $\mathfrak{a}_{P, \mathfrak{q}}:=\mathfrak{a}_{P} \cap \mathfrak{q}$ in $W=N_{K}\left(\mathfrak{a}_{\mathfrak{q}}\right) / Z_{K}\left(\mathfrak{a}_{\mathfrak{q}}\right)$. Here $N_{K}\left(\mathfrak{a}_{\mathfrak{q}}\right)$ and $Z_{K}\left(\mathfrak{a}_{\mathfrak{q}}\right)$ denote the normalizer of $\mathfrak{a}_{\mathfrak{q}}$ in $K$ and the centralizer of $\mathfrak{a}_{\mathfrak{q}}$ in $K$ respectively. Let $N_{K \cap H}\left(\mathfrak{a}_{\mathfrak{q}}\right)$ be the normalizer of $\mathfrak{a}_{\mathfrak{q}}$ in $K \cap H$. We denote by $W_{K \cap H}$ the image of $N_{K \cap H}\left(\mathfrak{a}_{\mathfrak{q}}\right)$ under the projection $N_{K}\left(\mathfrak{a}_{\mathfrak{q}}\right) \longrightarrow W$. Clearly $W_{K \cap H}$ is a subgroup of $W$. We fix a collection $\mathcal{W}_{P}$ of representatives for $W_{P} \backslash W / W_{K \cap H}$ contained in $N_{K}\left(\mathfrak{a}_{\mathfrak{q}}\right)$. We denote by $P \backslash G / H$ the collection of $H$-orbits on $P \backslash G$ and by $(P \backslash G / H)_{\text {open }}$ the subset of open orbits. Then we can show that the set $P \backslash G / H$ is finite and that the union $\cup_{v \in \mathcal{W}_{P}} P v H$ is an open dense subset of $P \backslash G$.

For $v \in \mathcal{W}_{P}$, we set

$$
X_{P, v}:=M_{P} /\left(M_{P} \cap v H v^{-1}\right) .
$$

We see that $X_{P, v}$ is a reductive symmetric space. Let $\mathrm{DS}_{P, v}$ be the set of all discrete series representations of $M_{P}$ for $X_{P, v}$. We set

$$
{ }^{*} \mathfrak{a}_{P, \mathfrak{q}}:=\mathfrak{a}_{\mathfrak{q}} \cap \mathfrak{m}_{P}, \quad{ }^{*} A_{P, \mathfrak{q}}=\exp \left({ }^{*} \mathfrak{a}_{P, \mathfrak{q}}\right) .
$$
by

For $\left(\xi, \mathcal{H}_{\xi}\right) \in \widehat{M_{P}}$ and $v \in \mathcal{W}_{P}$, we define the finite dimensional Hilbert space $V(P, \xi, v)$

$$
V(P, \xi, v):=\left\{\begin{array}{cl}
\left(\mathcal{H}_{\xi}^{-\infty}\right)^{M_{P} \cap v H v^{-1}} & \text { if } \xi \in \mathrm{DS}_{P, v} \\
0 & \text { otherwise }
\end{array}\right.
$$


Here $\mathcal{H}_{\xi}^{-\infty}$ denotes the continuous linear dual of the conjugate Frechét space $\overline{\mathcal{H}_{\xi}^{\infty}}$, that is, the set of all generalized vectors of $\mathcal{H}_{\xi}$ and $\left(\mathcal{H}_{\xi}^{-\infty}\right)^{M_{P} \cap v H v^{-1}}$ is the space of $\left(M_{P} \cap v H v^{-1}\right)$ fixed vectors in $\mathcal{H}_{\xi}^{-\infty}$.

Let $C^{\infty}(P: \xi: \lambda)$ be the space of smooth functions $f: G \longrightarrow \mathcal{H}_{\xi}$ satisfying the transformation law (4.1). Let $C^{-\infty}(P: \xi: \lambda)$ be the space of all generalized vectors of $C^{\infty}(P: \xi: \lambda)$. We denote by $C^{-\infty}(P: \xi: \lambda)^{H}$ the space of $H$-fixed generalized vectors for $\pi_{P, \xi, \lambda}$. Let $C^{\infty}(K: \xi)$ denote the space of smooth functions $\varphi: K \longrightarrow \mathcal{H}_{\xi}$ satisfying the transformation law

$$
\varphi(m k)=\xi(m) \varphi(k), \quad k \in K, m \in K \cap P=K \cap M_{P} .
$$

We denote by $C^{-\infty}(K: \xi)$ the space of all generalized vectors of $C^{\infty}(K: \xi)$.

If $\left(\xi, \mathcal{H}_{\xi}\right) \in \widehat{M_{P}}$, we define $V(P, \xi)$ to be the formal direct sum

$$
V(P, \xi):=\oplus_{v \in \mathcal{W}_{P}} V(P, \xi, v) .
$$

If $\eta \in V(P, \xi)$, the $\eta_{v}$ denotes its component in $V(P, \xi, v)$.

Definition. Let $\eta \in V(P, \xi)$. For $\lambda \in\left(\mathfrak{a}_{P, \mathfrak{q}}\right)_{\mathbb{C}}^{*}$ with $-\left(\operatorname{Re} \lambda+\rho_{P}\right)$ strictly $P$-dominant (i.e., $-<\operatorname{Re} \lambda+\rho_{P}, \alpha>>0$ for all $\left.\alpha \in \Sigma(P)\right)$, we define the function

$$
j(P: \xi: \lambda: \eta): G \longrightarrow \mathcal{H}_{\xi}^{-\infty}
$$

by

$$
j(P: \xi: \lambda: \eta)(\text { manvh })=\left\{\begin{array}{cl}
a^{\lambda+\rho_{P}} \xi(m) \eta_{v} & \text { if manvh } \in \cup_{v \in \mathcal{W}_{P}} P v H \\
0 & \text { otherwise. }
\end{array}\right.
$$

We see that $j(P: \xi: \lambda: \eta)$ is an element of $C^{-\infty}(P: \xi: \lambda)^{H}$ and has an analytic continuation to $\left(\mathfrak{a}_{P, \mathfrak{q}}\right)_{\mathbb{C}}^{*}$. If $\lambda$ is regular, the map

$$
j(P: \xi: \lambda): V(P, \xi) \longrightarrow C^{-\infty}(P: \xi: \lambda)^{H}
$$

defined by

$$
j(P: \xi: \lambda)(\eta)=j(P: \xi: \lambda: \eta)
$$

is an injective homomorphism. We define

$$
\operatorname{DS}_{P}:=\left\{\xi \in \widehat{M_{P}} \mid V(P, \xi) \neq 0\right\} .
$$

Now we will formulate the Plancherel formula for $G / H$ representation theoretically. Let $\mathcal{P}_{\sigma}$ be the set of all $\sigma \theta$-stable parabolic subgroups of $G$ containing $A_{\mathfrak{q}}$. Two parabolic subgroups $P, Q \in \mathcal{P}_{\sigma}$ are said to be $\sigma$-associated if their $\sigma$-split components $\mathfrak{a}_{P, \mathfrak{q}}$ and $\mathfrak{a}_{Q, \mathfrak{q}}$ are conjugate under the Weyl group $W$. If $P$ and $Q$ are $\sigma$-associated, we denote $P \sim Q$. It is easily seen that $\sim$ is an equivalence on $\mathcal{P}_{\sigma}$. Let $\mathbb{P}_{\sigma}$ be a set of representatives in $\mathcal{P}_{\sigma}$ for the equivalence classes $\mathcal{P}_{\sigma} / \sim$.

\section{Step I. The Fourier transform.}

Let $f \in C_{c}^{\infty}(G / H)$. The Fourier transform $\widehat{f}$ of $f$ is defined by

$$
\widehat{f}(P: \xi: \lambda)=\int_{G / H} f(x) \pi_{P, \xi, \lambda}(x) j(P: \xi: \lambda) d x
$$


for $P \in \mathcal{P}_{\sigma}, \xi \in \mathrm{DS}_{P}$ and generic $\lambda \in i \mathfrak{a}_{P, \mathfrak{q}}^{*}$. We can show that for given fixed $P \in \mathcal{P}_{\sigma}$ and $\xi \in \mathrm{DS}_{P}$, the map

$$
f \mapsto \widehat{f}(P: \xi: \lambda)
$$

intertwines the regular representation $\pi_{H}$ of $G$ and the representation $1 \otimes \pi_{P, \xi, \lambda}$ for generic $\lambda \in \mathfrak{a}_{P, \mathfrak{q}}^{*}$

\section{Step II. The Plancherel measure.}

Let $P, Q \in \mathcal{P}_{\sigma}$ such that $A_{P, \mathfrak{q}}=A_{Q, \mathfrak{q}}$. Then $M_{P}=M_{Q}, A_{P}=A_{Q}$, and $\mathrm{DS}_{P}=\mathrm{DS}_{Q}$. Let $\bar{P}$ and $\bar{Q}$ be the opposite parabolic subgroups of $P$ and $Q$ respectively. Then $\bar{P}$ and $\bar{Q}$ have the Langlands decompositions

$$
\bar{P}=M_{P} A_{P} \bar{N}_{P} \quad \text { and } \quad \bar{Q}=M_{Q} A_{Q} \bar{N}_{Q}
$$

We write

$$
\Sigma(Q: P)=\left\{\alpha \in \Sigma \mid \mathfrak{g}_{\alpha} \subset \overline{\mathfrak{n}}_{Q} \cap \mathfrak{n}_{P}\right\}
$$

for $\lambda \in\left(\mathfrak{a}_{P, \mathfrak{q}}\right)_{\mathbb{C}}^{*}$ with $<\operatorname{Re} \lambda, \alpha>$ sufficiently large for each $\alpha \in \Sigma(Q: P)$, then the following integral

$$
A(Q: P: \xi: \lambda) f(x):=\int_{N_{Q} \cap \bar{N}_{P}} f(n x) d n
$$

converges absolutely for $f \in C^{\infty}(P: \xi: \lambda)$, where $d n$ is a suitably normalized Haar measure of $N_{Q} \cap \bar{N}_{P}$. The above operator

$$
A(Q: P: \xi: \lambda): C^{\infty}(P: \xi: \lambda) \longrightarrow C^{\infty}(Q: \xi: \lambda)
$$

is an intertwining operator between $\pi_{P, \xi, \lambda}$ and $\pi_{Q, \xi, \lambda}$, and has an analytic continuation to $\left(\mathfrak{a}_{P, \mathfrak{q}}\right)_{\mathbb{C}}^{*}$. The operator $A(Q: P: \xi: \lambda)$ is called the standard intertwining operator .

For any $P \in \mathcal{P}_{\sigma}$, we put

$$
\mathfrak{a}_{P, \mathfrak{q}}^{* \mathrm{reg}}:=\left\{\lambda \in \mathfrak{a}_{P, \mathfrak{q}}^{*} \mid<\lambda, \alpha>\neq 0 \text { for all } \alpha \in \Sigma(P)\right\} .
$$

According to Harish-Chandra's results, $\pi_{P, \xi, \lambda}$ is an irreducible unitary representation of $G$ when $\xi \in \operatorname{DS}_{P}$ and $\lambda \in i \mathfrak{a}_{P, \mathfrak{q}}^{* \text { reg }}$. The standard intertwining operator $A(Q: P: \xi: \lambda)$ has an adjoint

$$
A(Q: P: \xi:-\bar{\lambda})^{*}: C^{-\infty}(Q: \xi: \lambda) \longrightarrow C^{-\infty}(P: \xi: \lambda)
$$

which depends meromorphically on $\lambda \in\left(\mathfrak{a}_{P, \mathfrak{q}}\right)_{\mathbb{C}}^{*}$ and is equal to the continuous linear extension of $A(P: Q: \xi: \lambda)$ which will be also denoted by the same notation $A(P: Q: \xi: \lambda)$. The operator

$$
A(\bar{P}: P: \xi:-\bar{\lambda})^{*} \circ A(\bar{P}: P: \xi: \lambda)
$$

is a $G$-intertwining operator from $C^{\infty}(P: \xi: \lambda)$ to $C^{\infty}(P: \xi: \lambda)$ for generic $\lambda \in i \mathfrak{a}_{P, \mathfrak{q}}^{*}$. By the above statement, the operator (4.5) is a scalar multiple of the identity operator

$$
\eta(P: \xi: \lambda) I
$$

such that $\eta(P: \eta: \cdot):\left(\mathfrak{a}_{P, \mathfrak{q}}\right)_{\mathbb{C}}^{*} \longrightarrow \mathbb{C}$ is a meromorphic function. We now define the measure $d \mu_{P, \xi}$ on $i \mathfrak{a}_{P, \mathfrak{q}}^{*}$ by

$$
d \mu_{P, \xi}(\lambda):=\frac{1}{\eta(P: \xi: \lambda)} d \mu_{P}(\lambda)
$$

where $d \mu_{P}$ is a suitable Lebesque measure on $i \mathfrak{a}_{P, \mathfrak{q}}^{*}$. 
From now on, $W_{P}^{*}\left(\right.$ resp. $\left.W_{P}\right)$ denotes the normalizer (resp. centralizer) of $\mathfrak{a}_{P, \mathfrak{q}}$ in the Weyl group $W$. We define the group

$$
W\left(\mathfrak{a}_{P, \mathfrak{q}}\right):=W_{P}^{*} / W_{P} .
$$

van den Ban and Schlichlichtkrull [12] obtained the beautiful Plancherel formula.

Plancherel Formula A [12]: Let $f \in C_{c}^{\infty}(G / H)$. Then

$$
\|f\|_{L^{2}(G / H)}^{2}=\sum_{P \in \mathbb{P}_{\sigma}}\left[W: W_{P}^{*}\right] \sum_{\xi \in \mathrm{DS}_{P}} \int_{i \mathfrak{a}_{P, \mathfrak{q}}^{*}}\|\widehat{f}(P: \xi: \lambda)\|^{2} d \mu_{P, \xi}(\lambda) .
$$

We now describe the Plancherel formula (4.8) in terms of representations of $G$. For $P \in \mathcal{P}_{\sigma}$ and $\xi \in \mathrm{DS}_{P}$, we define the Hilbert space

$$
\mathcal{H}(P, \xi):=\overline{V(P, \xi)} \otimes L^{2}(K: \xi),
$$

equipped with the tensor product inner product. In addition, we let

$$
\mathcal{L}^{2}(P, \xi):=L^{2}\left(i \mathfrak{a}_{P, \mathfrak{q}}^{*}, \mathcal{H}(P, \xi),\left[W: W_{P}^{*}\right] d \mu_{P, \xi}\right)
$$

be the space of square integrable functions from $i \mathfrak{a}_{P, \mathfrak{q}}^{*}$ to $\mathcal{H}(P, \xi)$ for the measure $[W$ : $\left.W_{P}^{*}\right] d \mu_{P, \xi}$. We define the representation $\pi_{P, \xi}$ of $G$ on $\mathcal{L}^{2}(P, \xi)$ by

$$
\left(\pi_{P, \xi}(g) \varphi\right)(\lambda):=\left[1 \otimes \pi_{P, \xi, \lambda}(g)\right] \varphi(\nu), \quad g \in G, \varphi \in \mathcal{L}^{2}(P, \xi), \lambda \in i \mathfrak{a}_{P, \mathfrak{q}}^{*} .
$$

$\pi_{P, \xi}$ is realized as the following direct integral

$$
\pi_{P, \xi} \cong \int_{i \mathfrak{a}_{P, \mathfrak{q}}^{*}} 1 \frac{}{V(P, \xi)} \otimes \pi_{P, \xi, \lambda}\left[W: W_{P}^{*}\right] d \mu_{P, \xi}(\lambda) .
$$

We define $\left(\pi_{P}, \mathcal{L}^{2}(P)\right)$ as the Hilbert direct sum of the unitary representations $\left(\pi_{P, \xi}, \mathcal{L}^{2}(P, \xi)\right)$, $\xi \in \mathrm{DS}_{P}$. Finally we define the representation $\left(\pi, \mathcal{L}^{2}\right)$ as the unitary direct sum of the representations $\left(\pi_{P}, \mathcal{L}^{2}(P)\right), P \in \mathbb{P}_{\sigma}$. Therefore we get

$$
\pi \cong \oplus_{P \in \mathbb{P}_{\sigma}} \hat{\oplus}_{\xi \in \mathrm{DS}_{P}} \pi_{P, \xi}
$$

For $P \in \mathbb{P}_{\sigma}$, we have a unitary representation of $W_{P}^{*}$ on $\mathcal{L}^{2}$ commuting with $\pi$. The space of $W_{P}^{*}$ on $\mathcal{L}^{2}$ is a closed $G$-invariant subspace of $\mathcal{L}^{2}$. The map $f \mapsto \widehat{f}$ extends to an isometry $\mathcal{F}$ from $L^{2}(G / H)$ into $\mathcal{L}^{2}$ intertwining $\pi_{H}$ with $\pi$. The image of the Fourier transform $\mathcal{F}$ is given by

$$
\operatorname{Im}(\mathcal{F})=\left(\mathcal{L}^{2}\right)^{W_{P}^{*}}
$$

The group $W\left(\mathfrak{a}_{P, \mathfrak{q}}\right)=W_{P}^{*} / W_{P}$ acts freely, but in general not transitively on the components of $\mathfrak{a}_{P, \mathfrak{q}}^{* \text { reg }}$. Let $\Omega_{P}$ be a fundamental domain for the action $W\left(\mathfrak{a}_{P, \mathfrak{q}}\right)$ on $i \mathfrak{a}_{P, \mathfrak{q}}^{* \text { reg }}$ consisting of connected components of $i \mathfrak{a}_{P, \mathfrak{q}}^{* \mathrm{reg}}$. Then, for each $P \in \mathcal{P}_{\sigma}$ and $\xi \in \mathrm{DS}_{P}$, we denote by $\mathcal{L}_{\Omega_{P}}^{2}(P, \xi)$ the closed $G$-invariant subspace of functions in $\mathcal{L}^{2}(P, \xi)$ that vanish almost everywhere outside $\Omega_{P}$. We define the closed $G$-invariant subspace

$$
\mathcal{L}_{0}^{2}:=\oplus_{P \in \mathbb{P}_{\sigma}} \hat{\oplus}_{\xi \in \mathrm{DS}_{P}} \mathcal{L}_{\Omega_{P}}^{2}(P, \xi) .
$$

The orthogonal projection of $\mathcal{L}^{2}$ onto $\mathcal{L}_{0}^{2}$ is denoted by $\varphi \mapsto \varphi_{0}$.

van den Ban and Schlichtkrull $[13,15]$ obtained the following results. 
Plancherel Formula B [13]: The map $f \mapsto(\mathcal{F} f)_{0}$ is an isometry from $L^{2}(G / H)$ onto $\mathcal{L}_{0}^{2}$ intertwining $\pi_{H}$ with $\pi$, establishing the Plancherel formula

$$
\pi_{H} \cong \oplus_{P \in \mathbb{P}_{\sigma}} \hat{\oplus}_{\xi \in \mathrm{DS}_{P}} \int_{\Omega_{P}} 1 \frac{}{V(P, \xi)} \otimes \pi_{P, \xi, \lambda}\left[W: W_{P}\right] d \mu_{P, \xi}(\lambda)
$$

In particular, for all $P \in \mathcal{P}_{\sigma}$ and $\xi \in \mathrm{DS}_{P}, \pi_{P, \xi, \lambda}$ occurs with multiplicity $\operatorname{dim} V(P, \xi)$ for almost all $\lambda \in i \mathfrak{a}_{P, \mathfrak{q}}^{*}$.

Next we provide a normalized version of the Plancherel formula (4.10). For $P \in \mathcal{P}_{\sigma}$ and $\xi \in \mathrm{DS}_{P}$, we define

$$
j^{\star}(P: \xi: \lambda):=A(\bar{P}: P: \xi: \lambda)^{-1} \circ j(\bar{P}: \xi: \lambda) .
$$

Regularity Theorem I [13]: The Hom $\left(V(P, \xi), C^{-\infty}(K: \xi)\right)$-valued function $j^{\star}(P: \xi: \cdot)$ is regular on $i \mathfrak{a}_{P, \mathfrak{q}}^{*}$.

For a minimal $\sigma$-parabolic subgroup $P$, the above theorem was solved by van den Ban and Schlichtkrull [8], and for a general $\sigma$-parabolic subgroup $P$, the above theorem was proved by Carmona and Delorme [19]. For $P \in \mathcal{P}_{\sigma}$ and $\xi \in \mathrm{DS}_{P}$, we now define the normalized Fourier transform $\widehat{f}_{\star}$ of a function $f \in C_{c}^{\infty}(G / H)$ by

$$
\widehat{f}_{\star}(P: \xi: \lambda):=\int_{G / H} f(x) \pi_{P, \xi, \lambda}(x) j^{\star}(P: \xi: \lambda) d x .
$$

According to Regularity Theorem, the function $\lambda \longrightarrow \widehat{f}_{\star}(P: \xi: \lambda)$ is analytic as a $\overline{V(P, \xi)} \otimes$ $C^{\infty}(K: \xi)$-valued function on $i \mathfrak{a}_{P, \mathfrak{q}}^{*}$. It is shown that for $P \in \mathcal{P}_{\sigma}, \xi \in \operatorname{DS}_{P}$ and $f \in$ $C_{c}^{\infty}(G / H)$

$$
\|\widehat{f}(P: \xi: \lambda)\|^{2} d \mu_{P, \xi}(\lambda)=\left\|\widehat{f}_{\star}(P: \xi: \lambda)\right\|^{2} d \mu_{P} .
$$

Let

$$
\mathcal{L}_{\star}^{2}(P, \xi):=L^{2}\left(i \mathfrak{a}_{P, \mathfrak{q}}^{*}, \mathcal{H}(P, \xi),\left[W: W_{P}^{*}\right] d \mu_{P}\right)
$$

be the space of square integrable functions from $i \mathfrak{a}_{P, \mathfrak{q}}^{*}$ to $\mathcal{H}(P, \xi)$ with respect to the measure $\left[W: W_{P}^{*}\right] d \mu_{P}$. We define the representation $\left(\pi_{\star}, \mathcal{L}_{\star}^{2}\right)$ as the unitary direct sum of the representation $\mathcal{L}_{\star}^{2}(P, \xi)$

$\left(P \in \mathbb{P}_{\sigma}, \xi \in \mathrm{DS}_{P}\right)$. In other words,

$$
\pi_{\star} \cong \oplus P \in \mathbb{P}_{\sigma} \hat{\oplus}_{\xi \in \mathrm{DS}_{P}} \mathcal{L}_{\star}^{2}(P, \xi) .
$$

We denote the continuous linear extension of $f \mapsto \widehat{f}_{\star}$ by $\mathcal{F}_{\star}: L^{2}(G / H) \longrightarrow \mathcal{L}_{\star}^{2}$. As before we define a unitary representation of $W\left(\mathfrak{a}_{P, \mathfrak{q}}\right)$ in $\mathcal{L}_{\star}^{2}$ commuting with $\pi_{\star}$. We fix fundamental domains $\Omega_{P} \subset i \mathfrak{a}_{P, \mathfrak{q}}^{* \text { reg }}$ for the action of $W\left(\mathfrak{a}_{P, \mathfrak{q}}\right)$, and define $\mathcal{L}_{\star, 0}^{2}$ in a similar way as $\mathcal{L}_{0}^{2}$. Then $\mathcal{L}_{\star, 0}^{2}$ is a closed $G$-invariant subspace of $\mathcal{L}_{\star}^{2}$. The orthogonal projection of $\mathcal{L}_{\star}^{2}$ onto $\mathcal{L}_{\star, 0}^{2}$ is denoted by $\varphi \mapsto \varphi_{0}$. The Plancherel formula (4.10) is essentially equivalent to the following normalized analogue.

Plancherel Formula $\mathbf{C}$ (A normalized version) [13, 15]: The map $f \mapsto\left(\mathcal{F}_{\star} f\right)_{0}$ is an isometry from $L^{2}(G / H)$ onto $\mathcal{L}_{\star, 0}^{2}$ intertwining $\pi_{H}$ with $\pi_{\star}$, establishing the Plancherel formula

$$
\pi_{H} \cong \oplus_{P \in \mathbb{P}_{\sigma}} \hat{\oplus}_{\xi \in \mathrm{DS}_{P}} \int_{i \Omega_{P}} 1 \frac{}{V(P, \xi)} \otimes \pi_{P, \xi, \lambda}\left[W: W_{P}\right] d \mu_{P}(\lambda)
$$


The Plancherel formula (4.12) is derived from the Plancherel formula for spherical functions and an application of a residue calculus.

We now describe the spherical Plancherel formula. Let $\left(\delta, V_{\delta}\right) \in \widehat{K}$ and let $L^{2}(G / H)_{\delta}$ be the subspace of $L^{2}(G / H)$ consisting of left $K$-finite functions of type $\delta$. We put $\tau=\tau_{\delta}:=$ $\delta^{*} \otimes 1$ and $V_{\tau}=V_{\delta}^{*} \otimes V_{\delta}$. We denote by $L^{2}(G / H: \tau)$ the space of $V_{\tau}$-valued functions $f: G / H \longrightarrow V_{\tau}$ satisfying the transformation behavior

$$
f(k x)=\tau(k) f(x), \quad k \in K, x \in G / H .
$$

Elements of $L^{2}(G / H: \tau)$ are called $\tau$-spherical functions or simply spherical functions. Similarly we can define $C_{c}^{\infty}(G / H: \tau), C^{\infty}(G / H: \tau)$ and $C(G / H: \tau)$. We observe that

$$
\begin{aligned}
L^{2}(G / H)_{\delta} & \cong \operatorname{Hom}_{K}\left(V_{\delta}, L^{2}(G / H)\right) \otimes V_{\delta} \\
& \cong\left(L^{2}(G / H) \otimes V_{\delta}^{*}\right)^{K} \otimes V_{\delta} \\
& \cong\left(L^{2}(G / H) \otimes V_{\tau}\right)^{K} \\
& \cong L^{2}(G / H: \tau) .
\end{aligned}
$$

The natural isomorphism

$$
\mathbb{S}: L^{2}(G / H)_{\delta} \longrightarrow L^{2}\left(G / H: \tau_{\delta}\right)
$$

is called the sphericalization of $L^{2}(G / H)_{\delta}$. Let $P \in \mathcal{P}_{\sigma}$ and $\xi \in \operatorname{DS}_{P}$. For generic $\lambda \in\left(\mathfrak{a}_{P, \mathfrak{q}}\right)_{\mathbb{D}}^{*}$, we define the map

$$
M_{P, \xi, \lambda}: \overline{V(P, \xi)} \otimes C^{\infty}(K: \xi) \longrightarrow C^{\infty}(G / H)
$$

by

$$
M_{P, \xi, \lambda}(\eta \otimes \varphi)(x):=<\varphi, \pi_{P, \xi,-\bar{\lambda}}(x) j^{\star}(P: \xi:-\bar{\lambda}) \eta>,
$$

where $\eta \otimes \varphi \in \overline{V(P, \xi)} \otimes C^{\infty}(K: \xi)$ and $x \in G / H$. We consider the matrix coefficient

$$
\mathbb{M}_{\xi, v}: \overline{V(P, \xi, v)} \otimes \mathcal{H}_{\xi} \longrightarrow L^{2}\left(X_{P, v}\right)
$$

defined by

$$
\mathbb{M}_{\xi, v}(\eta \otimes w)(m):=<\xi(m) \eta, w>,
$$

where $m \in M_{P}, \eta \otimes w \in \overline{V(P, \xi, v)} \otimes \mathcal{H}_{\xi}$. For $\xi \in \mathrm{DS}_{P}$, we set

$$
L^{2}\left(X_{P, v}\right)_{\xi}:=\mathbb{M}_{\xi, v}\left(\overline{V(P, \xi, v)} \otimes \mathcal{H}_{\xi}\right) .
$$

We note that $L^{2}\left(X_{P, v}\right)_{\xi} \neq 0$ if and only if $\xi \in \mathrm{DS}_{P}$.

We set

Then

$$
K_{P}:=K \cap M_{P} \quad \text { and } \quad \tau_{P}=\tau_{\delta, P}:=\left.\tau_{\delta}\right|_{K_{P}}
$$

We define

$$
L^{2}\left(X_{P, v}: \tau_{P}\right)=\left(L^{2}\left(X_{P, v}\right) \otimes V_{\tau}\right)^{K_{P}} .
$$

$$
L^{2}\left(X_{P, v}: \tau_{P}\right)_{\xi}:=\left(L^{2}\left(X_{P, v}\right)_{\xi} \otimes V_{\tau}\right)^{K_{P}} .
$$

We can show that there exists a canonical isometry

$$
\alpha_{\xi, \delta}: \overline{V(P, \xi)} \otimes C^{\infty}(K: \xi)_{\delta} \longrightarrow \oplus_{v \in \mathcal{W}_{P}} L^{2}\left(X_{P, v}: \tau_{P}\right)_{\xi},
$$

$\oplus$ denotes the formal direct sum of Hilbert spaces. Moreover $\overline{V(P, \xi)} \otimes C^{\infty}(K: \xi)_{\delta}$ is finite dimensional. 
Definition of Eisenstein integrals. Let $\psi \in \oplus_{v \in \mathcal{W}_{P}} L^{2}\left(X_{P, v}: \tau_{P}\right)_{\xi}$. For $\lambda \in\left(\mathfrak{a}_{P, \mathfrak{q}}\right)_{\mathbb{C}}^{*}$, we define the normalized Eisenstein integral $E^{0}(P: \psi: \lambda)$ by

$$
E^{0}(P: \psi: \lambda):=\mathbb{S}\left(M_{P, \xi,-\lambda}\left(\alpha_{\xi, \delta}^{-1}(\psi)\right)\right) \in L^{2}(G / H: \tau) .
$$

From now on, for any finite dimensional unitary representation $\left(\chi, V_{\chi}\right)$ of $K$, we let $\mathcal{A}_{2}(G / H: \chi)$ be the space of smooth functions $f \in C^{\infty}(G / H: \chi)$ satisfying the following conditions

(a) $f \in L^{2}(G / H: \chi)$;

(b) $\mathbb{D}(G / H) f$ is finite dimensional.

Here $\mathbb{D}(G / H)$ denotes the commutative algebra of all $G$-invariant differential operators on $G / H$. According to [36], $\mathcal{A}_{2}(G / H: \chi)$ is finite dimensional and is decomposed into the orthogonal direct sum

$$
\mathcal{A}_{2}(G / H: \chi)=\oplus_{\pi \in \operatorname{Disc}(G / H)} L^{2}(G / H: \chi)_{\pi} .
$$

In fact, the number of $\pi \in \operatorname{Disc}(G / H)$ such that $L^{2}(G / H: \chi)_{\pi} \neq 0$ is finite. We define the formal sum of Hilbert spaces

$$
\mathcal{A}_{2, P}:=\oplus_{v \in \mathcal{W}_{P}} \mathcal{A}_{2}\left(X_{P, v}: \tau_{P}\right) .
$$

By the formula (4.18) for $X_{P, v}\left(v \in \mathcal{W}_{P}\right), \mathcal{A}_{2, P}$ is decomposed into the orthogonal direct sum

$$
\mathcal{A}_{2, P}:=\oplus_{\xi \in \mathrm{DS}_{P}} \oplus_{v \in \mathcal{W}_{P}} \mathcal{A}_{2}\left(X_{P, v}: \tau_{P}\right)_{\xi} .
$$

For $\psi \in \mathcal{A}_{2, P}$, we define the normalized Eisenstein integral $E^{0}(P: \psi: \lambda)$ by

$$
E^{0}(P: \psi: \lambda):=\sum_{\xi \in \mathrm{DS}_{P}} E^{0}\left(P: \psi_{\xi}: \lambda\right)
$$

where $\psi_{\xi}$ is the component in $\mathcal{A}_{2, P, \xi}:=\oplus_{v \in \mathcal{W}_{P}} L^{2}\left(X_{P, v}: \tau_{P}\right)_{\xi}$.

Regularity Theorem II. The meromorphic function

$$
E^{0}(P: \psi: \cdot):\left(\mathfrak{a}_{P, \mathfrak{q}}\right)_{\mathbb{C}}^{*} \longrightarrow C^{\infty}(G / H: \tau)
$$

is regular on $i \mathfrak{a}_{P, \mathfrak{q}}^{*}$ for any $\psi \in \mathcal{A}_{2, P}$.

This regularity theorem is essentially equivalent to that for $J^{\star}(P: \xi: \cdot)$. The proof of the above regularity theorem is based on an asymptotic behavior of $E^{0}(P: \psi: \lambda)$ together with the Maass-Selberg relations among Harish-Chandra's $C$-functions.

For $\lambda \in\left(\mathfrak{a}_{P, \mathfrak{q}}\right)_{\mathbb{C}}^{*}$, we consider the map

$$
E^{0}(P: \lambda): G / H \longrightarrow \operatorname{Hom}\left(\mathcal{A}_{2, P}, V_{\tau}\right)
$$

defined by

$$
E^{0}(P: \lambda)(x) \psi=E^{0}(P: \lambda: x) \psi:=E^{0}(P: \psi: \lambda)(x) .
$$

We define the dual Eisenstein integral $E^{*}(P: \lambda: x)$ of $E^{0}(P: \lambda: x)$ by

$$
E^{*}(P: \lambda: x):=E^{0}(P:-\bar{\lambda}: x)^{*} \in \operatorname{Hom}\left(V_{\tau}, \mathcal{A}_{2, P}\right)
$$


for $x \in G / H$ and generic $\lambda \in\left(\mathfrak{a}_{P, \mathfrak{q}}\right)_{\mathbb{C}}^{*}$. For a given spherical function $f \in C_{c}^{\infty}(G / H: \tau)$, we define the spherical Fourier transform $\mathcal{F}_{P} f: i \mathfrak{a}_{P, \mathfrak{q}}^{*} \longrightarrow \mathcal{A}_{2, P}$ of $f$ by

$$
\mathcal{F}_{P} f(\lambda):=\int_{G / H} E^{*}(P: \lambda: x) f(x) d x .
$$

We let $\mathcal{S}\left(i \mathfrak{a}_{P, \mathfrak{q}}^{*}\right)$ be the space of Schwartz functions on $i \mathfrak{a}_{P, \mathfrak{q}}^{*}$ and let $\mathcal{C}(G / H)$ be the $L^{2}$-Schwartz space of $G / H$ consisting of smooth functions $f: G / H \longrightarrow \mathbb{C}$ such that

$$
\left(1+\log _{H}\right)^{n} X f \in L^{2}(G / H)
$$

for any $X \in U(\mathfrak{g})$ and any $n \in \mathbb{Z}^{+}$. Here $\log _{H}: G \longrightarrow[0, \infty)$ is a function on $G / H$ defined by $\log _{H}(k a h)=\|\log a\|(k \in K, a \in A, h \in H)$ and $U(\mathfrak{g})$ denotes the universal enveloping algebra of $\mathfrak{g}$. According to [4], we get the following facts :

$(\mathrm{SF} 1)<\widehat{f}_{\star}(P: \xi: \lambda), T>=<\mathcal{F} \mathbb{S}(f)(-\lambda), \alpha_{\xi, \delta}(T)>$ for $f \in C_{c}^{\infty}(G / H: \tau), T \in$ $\overline{V(P, \xi)} \otimes L^{2}(K: \xi)$ and all $\lambda \in i \mathfrak{a}_{P, \mathfrak{q}}^{*}$

(SF2) For $P \in \mathcal{P}_{\sigma}, \mathcal{F}_{P}$ maps $C_{c}^{\infty}(G / H: \tau)$ into $\mathcal{S}\left(i \mathfrak{a}_{P, \mathfrak{q}}^{*}\right) \otimes \mathcal{A}_{2, P}$.

(SF3) $\mathcal{F}_{P}$ extends to a continuous operator from $\mathcal{C}(G / H: \tau)$ to $\mathcal{S}\left(i \mathfrak{a}_{P, \mathfrak{q}}^{*}\right) \otimes \mathcal{A}_{2, P}$.

According to (SF3), we can define the wave packet operator $\mathcal{T}_{P}: \mathcal{S}\left(i \mathfrak{a}_{P, \mathfrak{q}}^{*}\right) \otimes \mathcal{A}_{2, P} \longrightarrow$ $C^{\infty}(G / H: \tau)$ by

$$
\mathcal{T}_{P} \varphi(x):=\int_{i \mathfrak{a}_{P, \mathfrak{q}}^{*}} E^{0}(P: \lambda: x) \varphi(\lambda) d \mu_{P}(\lambda) .
$$

It was proved by van den Ban and Schlichtkrull [7,9] that for $f \in C_{c}^{\infty}(G / H: \tau)$

$$
f=\sum_{P \in \mathbb{P}_{\sigma}}\left[W: W_{P}^{*}\right] \mathcal{T}_{P}\left(\mathcal{F}_{P} f\right)
$$

and that for associated $P, Q \in \mathcal{P}_{\sigma}$,

$$
\mathcal{T}_{P} \circ \mathcal{F}_{P}=\mathcal{T}_{Q} \circ \mathcal{F}_{Q} \quad \text { on } \mathcal{C}(G / H: \tau) .
$$

The following asymptotic behavior of the Eisenstein integral is obtained.

Theorem 4.1. Let $P, Q \in \mathcal{P}_{\sigma}$ be associated. Let

$$
W\left(\mathfrak{a}_{Q, \mathfrak{q}} \mid \mathfrak{a}_{P, \mathfrak{q}}\right):=\left\{\left.s\right|_{\mathfrak{a}_{P, \mathfrak{q}}} \mid s \in W, s\left(\mathfrak{a}_{P, \mathfrak{q}}\right) \subset \mathfrak{a}_{Q, \mathfrak{q}}\right\} .
$$

Then there are uniquely determined functions, called the $C$-functions

$$
C_{Q \mid P}^{0}(s: \cdot):\left(\mathfrak{a}_{P, \mathfrak{q}}\right)_{\mathbb{C}}^{*} \longrightarrow \operatorname{Hom}\left(\mathcal{A}_{2, P}, \mathcal{A}_{2, Q}\right), \quad s \in W\left(\mathfrak{a}_{Q, \mathfrak{q}} \mid \mathfrak{a}_{P, \mathfrak{q}}\right)
$$

such that

$$
E^{0}(P: \lambda: \operatorname{mav}) \psi \sim \sum_{s \in W\left(\mathfrak{a}_{Q, \mathfrak{q}} \mid \mathfrak{a}_{P, \mathfrak{q}}\right)} a^{s \lambda-\rho_{Q}}\left[C_{Q \mid P}^{0}(s: \lambda) \psi\right]_{v}(m)
$$

as $a \longrightarrow \infty$ in $A_{Q, \mathfrak{q}}^{+}$for all $\lambda \in i \mathfrak{a}_{P, \mathfrak{q}}^{*}, v \in \mathcal{W}_{Q}, m \in X_{Q, v}$ and $\psi \in \mathcal{A}_{2, P}$. Here $s \lambda:=\lambda \circ s^{-1}$.

Maass-Selberg Relations : Let $P, Q \in \mathcal{P}_{\sigma}$ be associated and let $s \in W\left(\mathfrak{a}_{Q, \mathfrak{q}} \mid \mathfrak{a}_{P, \mathfrak{q}}\right)$. Then

$$
C_{Q \mid P}^{0}(s:-\bar{\lambda})^{*} \circ C_{Q \mid P}^{0}(s: \lambda)=I .
$$


In particular, if $\lambda \in i \mathfrak{a}_{P, \mathfrak{q}}^{*}$, then $C_{Q \mid P}^{0}(s: \lambda)$ is unitary.

For the group case, the above result is due to Harish-Chandra [28], for $P$ minimal, it was obtained by van den Ban [4] and for general $P$, it was due to Carmona and Delorme [19].

The $C$-functions $C_{Q \mid P}^{0}(s: \lambda)$ have the following properties.

(C1) $C_{P \mid P}^{0}(1: \lambda)=I$.

(C2) For each $s \in W\left(\mathfrak{a}_{Q, \mathfrak{q}} \mid \mathfrak{a}_{P, \mathfrak{q}}\right)$,

$$
E^{0}(P: \lambda: x)=E^{0}(Q: s \lambda: x) \circ C_{Q \mid P}^{0}(s: \lambda)
$$

for any $x \in G / H$ and $\lambda \in\left(\mathfrak{a}_{Q, \mathfrak{q}}\right)_{\mathbb{C}}^{*}$.

(C3) Let $P, Q, R \in \mathcal{P}_{\sigma}$ be associated and $s \in W\left(\mathfrak{a}_{Q, \mathfrak{q}} \mid \mathfrak{a}_{P, \mathfrak{q}}\right)$. Then

$$
C_{R \mid P}^{0}(t s: \lambda)=C_{R \mid Q}^{0}(t: s \lambda) \circ C_{Q \mid P}^{0}(s: \lambda)
$$

as a $\operatorname{Hom}\left(\mathcal{A}_{2, P}, \mathcal{A}_{2, R}\right)$-valued identity of meromorphic functions on $\left(\mathfrak{a}_{Q, \mathfrak{q}}\right)_{\mathbb{C}}^{*}$.

(C4) Let $P, Q \in \mathcal{P}_{\sigma}$ be associated. Then for every $f \in \mathcal{C}(G / H: \tau)$ and $s \in W\left(\mathfrak{a}_{Q, \mathfrak{q}} \mid \mathfrak{a}_{P, \mathfrak{q}}\right)$,

$$
\mathcal{F}_{Q} f(s \lambda)=C_{Q \mid P}^{0}(s: \lambda) \mathcal{F}_{P} f(\lambda), \quad \lambda \in i \mathfrak{a}_{P, \mathfrak{q}}^{*}
$$

The functional equation (4.28) was originally obtained by Harish-Chandra [28] for the group case. For $P, Q$ minimal, (4.28) is due to van den Ban [4] and for general $P, Q$, it is due to Carmona and Delorme [19]. Recently van den Ban and Schlichtkrull gave another proof of (4.28) based on the principle of induction [11] involving the idea that both sides of (4.28) are eigenfunctions depending meromorphically on $\lambda \in\left(\mathfrak{a}_{Q, \mathfrak{q}}\right)_{\mathbb{C}}^{*}$. The formula (4.29) follows from the formula (4.28) by comparing the coefficients of $a^{t s \lambda-\rho_{R}}$ in the asymptotic expansions along $A_{R, \mathfrak{q}}^{+}$for $v \in \mathcal{W}_{R}$. The formula (4.30) follows from the following functional equation

$$
C_{Q \mid P}^{0}(s: \lambda) \circ E^{*}(P: \lambda: x)=E^{*}(Q: s \lambda: x)
$$

obtained from the equation (4.28) and Maass-Selberg relations.

Motivated by the formula (4.30) with $P=Q$, we define

$$
\left[\mathcal{S}\left(i \mathfrak{a}_{P, \mathfrak{q}}^{*}\right) \otimes \mathcal{A}_{2, P}\right]^{W\left(\mathfrak{a}_{P, \mathfrak{q}}\right)}
$$

to be the subspace of $\mathcal{S}\left(i \mathfrak{a}_{P, \mathfrak{q}}^{*}\right) \otimes \mathcal{A}_{2, P}$ consisting of the functions $f$ satisfying the following transformation law

$$
f(s \lambda)=C_{P \mid P}^{0}(s: \lambda) f(\lambda), \quad \lambda \in i \mathfrak{a}_{P, \mathfrak{q}}^{*}, s \in W\left(\mathfrak{a}_{P, \mathfrak{q}}\right) .
$$

Then we see that $\mathcal{F}_{P}$ maps $\mathcal{C}(G / H: \tau)$ into $\left[\mathcal{S}\left(i \mathfrak{a}_{P, \mathfrak{q}}^{*}\right) \otimes \mathcal{A}_{2, P}\right]^{W\left(\mathfrak{a}_{P, \mathfrak{q}}\right)}$.

van den Ban and Schlichtkrull [12] obtained the following.

The Plancherel formula for Spherical functions : The map $\mathcal{F}:=\sum_{P \in \mathbb{P}_{\sigma}} \mathcal{F}_{P}$ is a topological linear isomorphism from $\mathcal{C}(G / H: \tau)$ onto $\oplus_{P \in \mathbb{P}_{\sigma}}\left[\mathcal{S}\left(i \mathfrak{a}_{P, \mathfrak{q}}^{*}\right) \otimes \mathcal{A}_{2, P}\right]^{W\left(\mathfrak{a}_{P, \mathfrak{q}}\right)}$. The inverse of $\mathcal{F}$ is given by

$$
\mathcal{T}:=\oplus_{P \in \mathbb{P}_{\sigma}}\left[W: W_{P}^{*}\right] \mathcal{T}_{P}
$$


Moreover, for every $f \in C(G / H: \tau)$,

$$
\|f\|_{L^{2}(G / H: \tau)}^{2}=\oplus_{P \in \mathbb{P}_{\sigma}}\left[W: W_{P}^{*}\right]\left\|\mathcal{F}_{P} f\right\|_{L^{2}}^{2} .
$$

\section{An Example of a Homogeneous Space of Non-Reductive Type}

In this section, we present some results and survey some known results on the homogeneous space $\mathbb{H}_{n} \times \mathbb{C}^{(m, n)}$ of non-reductive type which is important arithmetically and geometrically.

\subsection{Invariant differential operators on the Siegel upper half plane.}

For a given fixed positive integer $n$, we let

$$
\mathbb{H}_{n}=\left\{\Omega \in \mathbb{C}^{(n, n)} \mid \Omega={ }^{t} \Omega, \quad \operatorname{Im} \Omega>0\right\}
$$

be the Siegel upper half plane of degree $n$ and let

$$
S p(n, \mathbb{R})=\left\{M \in \mathbb{R}^{(2 n, 2 n)} \mid{ }^{t} M J_{n} M=J_{n}\right\}
$$

be the symplectic group of degree $n$, where

$$
J_{n}=\left(\begin{array}{cc}
0 & I_{n} \\
-I_{n} & 0
\end{array}\right)
$$

$S p(n, \mathbb{R})$ acts on $\mathbb{H}_{n}$ transitively by

$$
M \cdot \Omega=(A \Omega+B)(C \Omega+D)^{-1},
$$

where $M=\left(\begin{array}{ll}A & B \\ C & D\end{array}\right) \in S p(n, \mathbb{R})$ and $\Omega \in \mathbb{H}_{n}$.

For brevity, we write $G=S p(n, \mathbb{R})$. We see that the stabilizer $K$ at $i I_{n}$ is given by

$$
K=\left\{\left(\begin{array}{cc}
A & B \\
-B & A
\end{array}\right) \in G \mid A+i B \in U(n)\right\}
$$

and hence the map

$$
G / K \longrightarrow \mathbb{H}_{n}, \quad g K \mapsto g \cdot\left(i I_{n}\right)
$$

is diffeomorphic (in fact, biholomorphic). Let $\mathfrak{g}$ be the Lie algebra of $G$ and let $\mathfrak{g}_{\mathbb{C}}$ be its complexification. The Killing form $B$ for $\mathfrak{g}$ is given by

$$
B(X, Y)=2(n+1) \operatorname{tr}(X Y), \quad X, Y \in \mathfrak{g} .
$$

Let $\theta$ be a Cartan involution of $G$ defined by

$$
\theta(g)={ }^{t} g^{-1}, \quad g \in G \text {. }
$$

$\mathfrak{g}$ has a Cartan decomposition

$$
\mathfrak{g}=\mathfrak{k} \oplus \mathfrak{p},
$$

where

and

$$
\mathfrak{k}=\left\{\left(\begin{array}{cc}
A & B \\
-B & A
\end{array}\right) \in \mathbb{R}^{(2 n, 2 n)} \mid A+{ }^{t} A=0, B={ }^{t} B\right\}
$$

$$
\mathfrak{p}=\left\{\left(\begin{array}{cc}
A & B \\
B & -A
\end{array}\right) \in \mathbb{R}^{(2 n, 2 n)} \mid A={ }^{t} A, B={ }^{t} B\right\} .
$$


We note that $\mathfrak{k}$ is the Lie algebra of $K$ and that $\mathfrak{k}$ and $\mathfrak{p}$ are orthogonal with respect to the Killing form $B$. The vector space $\mathfrak{p}$ is identified with the tangent space of $\mathbb{H}_{n}$ at $i I_{n}$. The correspondence

$$
\frac{1}{2}\left(\begin{array}{cc}
A & B \\
-B & A
\end{array}\right) \mapsto A+i B
$$

yields an isomorphism of $\mathfrak{p}$ onto the space $T_{n}$ of symmetric $n \times n$ complex matrices. The differential map $d \theta$ of $\theta$ extends complex linearly to $\mathfrak{g}_{\mathbb{C}}$. The $( \pm 1)$-eigenspaces of $d \theta$ are

$$
\mathfrak{k}_{\mathbb{C}}=\left\{\left(\begin{array}{cc}
A & B \\
-B & A
\end{array}\right) \in \mathbb{C}^{(2 n, 2 n)} \mid A+{ }^{t} A=0, B={ }^{t} B\right\}
$$

and

$$
\mathfrak{p}_{\mathbb{C}}=\left\{\left(\begin{array}{cc}
A & B \\
B & -A
\end{array}\right) \in \mathbb{C}^{(2 n, 2 n)} \mid A={ }^{t} A, B={ }^{t} B\right\}
$$

respectively. We observe that $\mathfrak{k}_{\mathbb{C}}$ and $\mathfrak{p}_{\mathbb{C}}$ are the complexifications of $\mathfrak{k}$ and $\mathfrak{p}$ respectively. $\mathfrak{p}_{\mathbb{C}}$ has the following decomposition

$$
\mathfrak{p}_{\mathbb{C}}=\mathfrak{p}_{+} \oplus \mathfrak{p}_{-}
$$

where

$$
\mathfrak{p}_{+}=\left\{\left(\begin{array}{cc}
X & i X \\
i X & -X
\end{array}\right) \in \mathbb{C}^{(2 n, 2 n)} \mid X={ }^{t} X\right\}
$$

and

$$
\mathfrak{p}_{-}=\left\{\left(\begin{array}{cc}
Y & -i Y \\
-i Y & -Y
\end{array}\right) \in \mathbb{C}^{(2 n, 2 n)} \mid Y={ }^{t} Y\right\} .
$$

We observe that $\mathfrak{p}_{+}$and $\mathfrak{p}_{-}$are abelian subalgebras of $\mathfrak{g}_{\mathbb{C}}$. It is seen that

$$
\left[\mathfrak{k}_{\mathbb{C}}, \mathfrak{k}_{\mathbb{C}}\right] \subset \mathfrak{k}_{\mathbb{C}}, \quad\left[\mathfrak{k}_{\mathbb{C}}, \mathfrak{p}_{\mathbb{C}}\right] \subset \mathfrak{p}_{\mathbb{C}}, \quad\left[\mathfrak{p}_{\mathbb{C}}, \mathfrak{p}_{\mathbb{C}}\right] \subset \mathfrak{k}_{\mathbb{C}}
$$

Since $\operatorname{Ad}(k) X=k X k^{-1}\left(k \in K, X \in \mathfrak{g}_{\mathbb{C}}\right)$, we get the relation

$$
\operatorname{Ad}(k) \mathfrak{p}_{+} \subset \mathfrak{p}_{+}, \quad \operatorname{Ad}(k) \mathfrak{p}_{-} \subset \mathfrak{p}_{-} .
$$

For instance, if $k=\left(\begin{array}{cc}A & B \\ -B & A\end{array}\right) \in K$, then

$$
\operatorname{Ad}(k)\left(\begin{array}{cc}
X & \pm i X \\
\pm i X & -X
\end{array}\right)=\left(\begin{array}{cc}
X^{\prime} & \pm i X^{\prime} \\
\pm i X^{\prime} & -X^{\prime}
\end{array}\right), \quad X={ }^{t} X
$$

where

$$
X^{\prime}=(A+i B) X^{t}(A+i B) .
$$

If we identify $\mathfrak{p}_{-}$with $T_{n}$, then the action of $K$ on $\mathfrak{p}_{-}$is compatible with the natural representation $\rho^{[1]}$ of $G L(n, \mathbb{C})$ on $T_{n}$ given by

$$
\rho^{[1]}(g)(X):=g X^{t} g, \quad g \in G L(n, \mathbb{C}), X \in T_{n} .
$$

For a coordinate $\Omega=\left(\omega_{\mu \nu}\right) \in \mathbb{H}_{n}$, we put

$$
\begin{aligned}
\Omega & =X+i Y, \quad X=\left(x_{\mu \nu}\right), \quad Y=\left(y_{\mu \nu}\right) \text { real, } \\
d \Omega & =\left(d \omega_{\mu \nu}\right), \quad d \bar{\Omega}=\left(d \bar{\omega}_{\mu \nu}\right), \\
\frac{\partial}{\partial \Omega}= & \left(\frac{1+\delta_{\mu \nu}}{2} \frac{\partial}{\partial \omega_{\mu \nu}}\right), \quad \frac{\partial}{\partial \bar{\Omega}}=\left(\frac{1+\delta_{\mu \nu}}{2} \frac{\partial}{\partial \bar{\omega}_{\mu \nu}}\right),
\end{aligned}
$$


where $\delta_{i j}$ denotes the Kronecker delta symbol. C. L. Siegel [41] introduced the symplectic metric

$$
d s_{n}^{2}=\operatorname{tr}\left(Y^{-1} d \Omega Y^{-1} d \bar{\Omega}\right)
$$

which is invariant under the action (5.1) of G. H. Maass [33] proved that the differential operator

$$
\Delta_{n}=4 \operatorname{tr}\left(Y^{t}\left(Y \frac{\partial}{\partial \bar{\Omega}}\right) \frac{\partial}{\partial \Omega}\right)
$$

is the Laplacian of $\mathbb{H}_{n}$ for the symplectic metric $d s_{n}^{2}$.

According to Harish-Chandra, we see that the algebra $\mathbb{D}\left(\mathbb{H}_{n}\right)$ of all $G$-invariant differential operators on $\mathbb{H}_{n}$ is generated by the $n$ algebraically independent commuting differential operators on $\mathbb{H}_{n}$. Here we note that $\operatorname{rank} G=n$. Therefore $\mathbb{D}\left(\mathbb{H}_{n}\right)$ is isomorphic to the polynomial ring $\mathbb{C}\left[x_{1}, \cdots, x_{n}\right]$. We see from (5.4) that $K$ acts on $T_{n}$ by

$$
k \cdot X=k X^{t} k, \quad k \in K, X \in T_{n} .
$$

This action induces naturally the action $\tau$ of $K$ on the polynomial algebra $\operatorname{Pol}\left(T_{n}\right)$ of $T_{n}$. We denote by $\operatorname{Pol}\left(T_{n}\right)^{K}$ the subalgebra of $\operatorname{Pol}\left(T_{n}\right)$ consisting of all $K$-invariants for the action $\tau$ of $K$. Then there is a canonical linear bijection

$$
\Psi: \operatorname{Pol}\left(T_{n}\right)^{K} \longrightarrow \mathbb{D}\left(\mathbb{H}_{n}\right)
$$

of $\operatorname{Pol}\left(T_{n}\right)^{K}$ onto $\mathbb{D}\left(\mathbb{H}_{n}\right)$. For more detail, we refer to [29], p. 287. In fact, $\operatorname{Pol}\left(T_{n}\right)^{K}$ is generated by

$$
p_{k}(X):=\sigma\left((X \bar{X})^{k}\right), \quad 1 \leq k \leq n
$$

and $\Psi\left(p_{1}(X)\right)=\Delta_{n}$. As far as I know, it seems that for general $n$, the invariant differential operators $\Psi\left(p_{k}(X)\right)(2 \leq k \leq n)$ were not written explicitly so far.

Let

$$
\mathbb{D}_{n}=\left\{W \in \mathbb{C}^{(n, n)} \mid W={ }^{t} W, I_{n}-\bar{W} W>0\right\}
$$

be the generalized unit disk of degree $n$. The Cayley transform $\Phi: \mathbb{D}_{n} \longrightarrow \mathbb{H}_{n}$ defined by

$$
\Phi(W):=i\left(I_{n}+W\right)\left(I_{n}-W\right)^{-1}, \quad W \in \mathbb{D}_{n}
$$

is a biholomorphic mapping of $\mathbb{D}_{n}$ onto $\mathbb{H}_{n}$ which gives the bounded realization of $\mathbb{H}_{n}$ by $\mathbb{D}_{n}$ (cf. [41],pp. 281-283). Let

$$
T=\frac{1}{\sqrt{2}}\left(\begin{array}{cc}
I_{n} & i I_{n} \\
i I_{n} & -i I_{n}
\end{array}\right)
$$

be the $2 n \times 2 n$ matrix represented by $\Phi$. Then we see that

$$
T^{-1} G T=\left\{\left(\frac{P}{Q} \frac{Q}{P}\right) \in S U(n, n) \mid{ }^{t} P \bar{P}-{ }^{t} \bar{Q} Q=I_{n},{ }^{t} P \bar{Q}={ }^{t} \bar{Q} P\right\} .
$$

For brevity, we set

$$
G_{*}=T^{-1} G T \text {. }
$$

If the case $n=1$, we note that $G_{*}=S U(1,1)$. If $n>1$, then $G_{*}$ is a proper subgroup of $S U(n, n)$. In fact, since ${ }^{t} T J_{n} T=-i J_{n}$, we get

$$
G_{*}=\left\{h \in S U(n, n) \mid{ }^{t} h J_{n} h=J_{n}\right\} .
$$


Let

$$
P^{+}=\left\{\left(\begin{array}{cc}
I_{n} & Z \\
0 & I_{n}
\end{array}\right) \mid Z={ }^{t} Z \in \mathbb{C}^{(n, n)}\right\}
$$

be the $P^{+}$-part of the complexification of $G_{*} \subset S U(n, n)$.

Since the Harish-Chandra decomposition of an element $\left(\left(\frac{P}{Q} \frac{Q}{P}\right)\right)$ in $G_{*}^{J}$ is

$$
\left(\begin{array}{cc}
\bar{P} & \frac{Q}{P}
\end{array}\right)=\left(\begin{array}{cc}
I_{n} & Q \bar{P}^{-1} \\
0 & I_{n}
\end{array}\right)\left(\begin{array}{cc}
P-Q \bar{P}^{-1} \bar{Q} & 0 \\
0 & \bar{P}
\end{array}\right)\left(\begin{array}{cc}
I_{n} & 0 \\
\bar{P}^{-1} \bar{Q} & I_{n}
\end{array}\right)
$$

the $P^{+}$-component of the following element

$$
\left(\begin{array}{cc}
P & Q \\
Q & P
\end{array}\right) \cdot\left(\begin{array}{cc}
I_{n} & W \\
0 & I_{n}
\end{array}\right), \quad W \in \mathbb{D}_{n}
$$

of the complexification of $G_{*}^{J}$ is given by

$$
\left(\left(\begin{array}{cc}
I_{n} & (P W+Q)(\bar{Q} W+\bar{P})^{-1} \\
0 & I_{n}
\end{array}\right)\right) .
$$

We note that $Q \bar{P}^{-1} \in \mathbb{D}_{n}$. We get the Harish-Chandra embedding of $\mathbb{D}_{n}$ into $P^{+}$(cf. [30], p. 155). Therefore we see that $G_{*}$ acts on $\mathbb{D}_{n}$ transitively by

$$
\left(\begin{array}{ll}
\frac{P}{Q} & \frac{Q}{P}
\end{array}\right) \cdot W=(P W+Q)(\bar{Q} W+\bar{P})^{-1},
$$

where $\left(\frac{P}{Q} \frac{Q}{P}\right) \in G_{*}$ and $W \in \mathbb{D}_{n}$. We can show that the action (5.11) of $G_{*}$ on $\mathbb{D}_{n}$ is compatible with the action (5.1) of $G$ through the Cayley transform $\Phi$.

For a coordinate $W \in \mathbb{D}_{n}$ with $W=\left(w_{\mu \nu}\right)$, we put

$$
d W=\left(d w_{\mu \nu}\right), \quad d \bar{W}=\left(d \bar{w}_{\mu \nu}\right)
$$

and

$$
\frac{\partial}{\partial W}=\left(\frac{1+\delta_{\mu \nu}}{2} \frac{\partial}{\partial w_{\mu \nu}}\right), \quad \frac{\partial}{\partial \bar{W}}=\left(\frac{1+\delta_{\mu \nu}}{2} \frac{\partial}{\partial \bar{w}_{\mu \nu}}\right) .
$$

Using the Cayley transform $\Phi$, we can see (cf. [41]) that

$$
d s_{*}^{2}=4 \operatorname{tr}\left(\left(I_{n}-W \bar{W}\right)^{-1} d W\left(I_{n}-\bar{W} W\right)^{-1} d \bar{W}\right)
$$

is a $G_{*}$-invariant Riemannian metric on $\mathbb{D}_{n}$ and H. Maass [33] showed that its Laplacian is given by

$$
\Delta_{*}=\sigma\left(\left(I_{n}-W \bar{W}\right)^{t}\left(\left(I_{n}-W \bar{W}\right) \frac{\partial}{\partial \bar{W}}\right) \frac{\partial}{\partial W}\right)
$$

\subsection{Invariant differential operators on the Siegel-Jacobi space.}

For two positive integers $n$ and $m$, we consider the Heisenberg group

$$
H_{\mathbb{R}}^{(n, m)}=\left\{(\lambda, \mu ; \kappa) \mid \lambda, \mu \in \mathbb{R}^{(m, n)}, \kappa \in \mathbb{R}^{(m, n)}, \kappa+\mu^{t} \lambda \text { symmetric }\right\}
$$

endowed with the following multiplication law

$$
(\lambda, \mu ; \kappa) \circ\left(\lambda^{\prime}, \mu^{\prime} ; \kappa^{\prime}\right)=\left(\lambda+\lambda^{\prime}, \mu+\mu^{\prime} ; \kappa+\kappa^{\prime}+\lambda^{t} \mu^{\prime}-\mu^{t} \lambda^{\prime}\right) .
$$


We let

$$
G^{J}:=S p(n, \mathbb{R}) \ltimes H_{\mathbb{R}}^{(n, m)} \quad \text { (the semidirect product) }
$$

be the Jacobi group endowed with the following multiplication law

$$
(M,(\lambda, \mu ; \kappa)) \cdot\left(M^{\prime},\left(\lambda^{\prime}, \mu^{\prime} ; \kappa^{\prime}\right)\right)=\left(M M^{\prime},\left(\tilde{\lambda}+\lambda^{\prime}, \tilde{\mu}+\mu^{\prime} ; \kappa+\kappa^{\prime}+\tilde{\lambda}^{t} \mu^{\prime}-\tilde{\mu}^{t} \lambda^{\prime}\right)\right)
$$

with $M, M^{\prime} \in S p(n, \mathbb{R}),(\lambda, \mu ; \kappa),\left(\lambda^{\prime}, \mu^{\prime} ; \kappa^{\prime}\right) \in H_{\mathbb{R}}^{(n, m)}$ and $(\tilde{\lambda}, \tilde{\mu})=(\lambda, \mu) M^{\prime}$. Then we get the canonical action of $G^{J}$ on $\mathbb{H}_{n} \times \mathbb{C}^{(m, n)}($ cf. $[43,44,45])$ defined by

$$
(M,(\lambda, \mu ; \kappa)) \cdot(\Omega, Z)=\left(M \cdot \Omega,(Z+\lambda \Omega+\mu)(C \Omega+D)^{-1}\right),
$$

where $M=\left(\begin{array}{ll}A & B \\ C & D\end{array}\right) \in S p(n, \mathbb{R}),(\lambda, \mu ; \kappa) \in H_{\mathbb{R}}^{(n, m)}$ and $(\Omega, Z) \in \mathbb{H}_{n} \times \mathbb{C}^{(m, n)}$. We note that the action (5.15) is transitive. The stabilizer $K^{J}$ of $G^{J}$ at $\left(i I_{n}, 0\right)$ is given by

$$
K^{J}=\left\{(k,(0,0 ; \kappa)) \mid k \in K, \kappa={ }^{t} \kappa \in \mathbb{R}^{(m, m)}\right\} .
$$

Therefore $\mathbb{H}_{n} \times \mathbb{C}^{(m, n)} \cong G^{J} / K^{J}$ is a homogeneous space of non-reductive type. The Lie algebra $\mathfrak{g}^{J}$ of $G^{J}$ has a decomposition

$$
\mathfrak{g}^{J}=\mathfrak{k}^{J}+\mathfrak{p}^{J},
$$

where

$$
\begin{aligned}
\mathfrak{k}^{J} & =\left\{(X,(0,0, \kappa)) \mid X \in \mathfrak{k}, \kappa={ }^{t} \kappa \in \mathbb{R}^{(m, m)}\right\} \\
\mathfrak{p}^{J} & =\left\{(Y,(P, Q, 0)) \mid Y \in \mathfrak{p}, P, Q \in \mathbb{R}^{(m, n)}\right\} .
\end{aligned}
$$

Thus the tangent space of the homogeneous space $\mathbb{H}_{n} \times \mathbb{C}^{(m, n)}$ at $\left(i I_{n}, 0\right)$ is identified with $\mathfrak{p}^{J}$. We define a complex structure $I^{J}$ on the tangent space $\mathfrak{p}^{J}$ of $\mathbb{H}_{n} \times \mathbb{C}^{(m, n)}$ at $\left(i I_{n}, 0\right)$ by

$$
I^{J}\left(\left(\begin{array}{cc}
Y & X \\
X & -Y
\end{array}\right),(P, Q)\right)=\left(\left(\begin{array}{cc}
X & -Y \\
-Y & -X
\end{array}\right),(Q,-P)\right)
$$

where $X={ }^{t} X \in \mathbb{R}^{(m, n)}$ and $Y={ }^{t} Y \in \mathbb{R}^{(m, n)}$. Identifying $\mathbb{R}^{(m, n)} \times \mathbb{R}^{(m, n)}$ with $\mathbb{C}^{(m, n)}$ via

$$
(P, Q) \mapsto i P+Q, \quad P, Q \in \mathbb{R}^{(m, n)},
$$

we may regard the complex structure $I^{J}$ as a real linear map

$$
I^{J}(X+i Y, Q+i P)=(-Y+i X,-P+i Q),
$$

where $X+i Y \in T_{n}$ and $Q+i P \in \mathbb{C}^{(m, n)}$. Obviously $I^{J}$ extends complex linearly on the complexification $\mathfrak{p}_{\mathbb{C}}^{J}$ of $\mathfrak{p}^{J}$. Then $\mathfrak{p}_{\mathbb{C}}^{J}$ has a decomposition

$$
\mathfrak{p}_{\mathbb{C}}^{J}=\mathfrak{p}_{+}^{J} \oplus \mathfrak{p}_{-}^{J},
$$

where $\mathfrak{p}_{+}^{J}\left(\right.$ resp. $\left.\mathfrak{p}_{-}^{J}\right)$ denotes the $(+i)$-eigenspace (resp. $(-i)$-eigenspace) of $I^{J}$. Precisely, both $\mathfrak{p}_{+}^{J}$ and $\mathfrak{p}_{-}^{J}$ are given by

$$
\mathfrak{p}_{+}^{J}=\left\{\left(\left(\begin{array}{cc}
X & i X \\
i X & -X
\end{array}\right),(P, i P)\right) \mid X={ }^{t} X \in \mathbb{C}^{(n, n)}, P \in \mathbb{C}^{(m, n)}\right\}
$$

and

$$
\mathfrak{p}_{-}^{J}=\left\{\left(\left(\begin{array}{cc}
X & -i X \\
-i X & -X
\end{array}\right),(P,-i P)\right) \mid X={ }^{t} X \in \mathbb{C}^{(n, n)}, P \in \mathbb{C}^{(m, n)}\right\}
$$


Let $\mathfrak{k}^{J}$ be the Lie algebra of $K^{J}$ and let $\mathfrak{k}_{\mathbb{C}}^{J}$ be its complexification. We can see that

$$
\left[\mathfrak{k}^{J}, \mathfrak{k}^{J}\right] \subset \mathfrak{k}^{J}, \quad\left[\mathfrak{k}^{J}, \mathfrak{p}^{J}\right] \subset \mathfrak{p}^{J}
$$

and

$$
\left[\mathfrak{k}_{\mathbb{C}}^{J}, \mathfrak{p}_{+}^{J}\right] \subset \mathfrak{p}_{+}^{J}, \quad\left[\mathfrak{k}_{\mathbb{C}}^{J}, \mathfrak{p}_{-}^{J}\right] \subset \mathfrak{p}_{-}^{J} .
$$

The complexification $\mathfrak{g}_{\mathbb{C}}^{J}$ of $\mathfrak{g}^{J}$ has a decomposition $\mathfrak{g}_{\mathbb{C}}^{J}=\mathfrak{k}_{\mathbb{C}}^{J}+\mathfrak{p}_{\mathbb{C}}^{J}$.

For brevity, we write $\mathbb{H}_{n, m}:=\mathbb{H}_{n} \times \mathbb{C}^{(m, n)}$. For a coordinate $(\Omega, Z) \in \mathbb{H}_{n} \times \mathbb{C}^{(m, n)}$ with $\Omega=\left(\omega_{\mu \nu}\right)$ and $Z=\left(z_{k l}\right)$, we put $d \Omega, d \bar{\Omega}, \frac{\partial}{\partial \Omega}, \frac{\partial}{\partial \bar{\Omega}}$ as before and set

$$
\begin{aligned}
& Z=U+i V, \quad U=\left(u_{k l}\right), \quad V=\left(v_{k l}\right) \text { real, } \\
& d Z=\left(d z_{k l}\right), \quad d \bar{Z}=\left(d \bar{z}_{k l}\right), \\
& \frac{\partial}{\partial \Omega}=\left(\frac{1+\delta_{\mu \nu}}{2} \frac{\partial}{\partial \omega_{\mu \nu}}\right), \quad \frac{\partial}{\partial \bar{\Omega}}=\left(\frac{1+\delta_{\mu \nu}}{2} \frac{\partial}{\partial \bar{\omega}_{\mu \nu}}\right), \\
& \frac{\partial}{\partial Z}=\left(\begin{array}{ccc}
\frac{\partial}{\partial z_{11}} & \cdots & \frac{\partial}{\partial z_{m 1}} \\
\vdots & \ddots & \vdots \\
\frac{\partial}{\partial z_{1 n}} & \cdots & \frac{\partial}{\partial z_{m n}}
\end{array}\right), \quad \frac{\partial}{\partial \bar{Z}}=\left(\begin{array}{ccc}
\frac{\partial}{\partial \bar{z}_{11}} & \cdots & \frac{\partial}{\partial \bar{z}_{m 1}} \\
\vdots & \ddots & \vdots \\
\frac{\partial}{\partial \bar{z}_{1 n}} & \cdots & \frac{\partial}{\partial \bar{z}_{m n}}
\end{array}\right) .
\end{aligned}
$$

In [48], the author proved the following theorems.

Theorem 5.1. The following metric

$$
\begin{array}{r}
d s_{n, m}^{2}=\operatorname{tr}\left(Y^{-1} d \Omega Y^{-1} d \bar{\Omega}\right)+\operatorname{tr}\left(Y^{-1 t} V V Y^{-1} d \Omega Y^{-1} d \bar{\Omega}\right) \\
+\operatorname{tr}\left(Y^{-1 t}(d Z) d \bar{Z}\right) \\
-\operatorname{tr}\left(V Y^{-1} d \Omega Y^{-1 t}(d \bar{Z})+V Y^{-1} d \bar{\Omega} Y^{-1 t}(d Z)\right)
\end{array}
$$

is a Riemannian metric on $\mathbb{H}_{n, m}$ which is invariant under the action (5.15) of the Jacobi group $G^{J}$. The Laplacian $\Delta_{n, m}$ of $\left(\mathbb{H}_{n, m}, d s_{n, m}^{2}\right)$ is given by

$$
\begin{aligned}
\Delta_{n, m}=\quad & 4 \operatorname{tr}\left(Y^{t}\left(Y \frac{\partial}{\partial \bar{\Omega}}\right) \frac{\partial}{\partial \Omega}\right)+4 \operatorname{tr}\left(Y \frac{\partial}{\partial Z}{ }^{t}\left(\frac{\partial}{\partial \bar{Z}}\right)\right) \\
+ & 4 \operatorname{tr}\left(V Y^{-1 t} V^{t}\left(Y \frac{\partial}{\partial \bar{Z}}\right) \frac{\partial}{\partial Z}\right) \\
+4 & \operatorname{tr}\left(V^{t}\left(Y \frac{\partial}{\partial \bar{\Omega}}\right) \frac{\partial}{\partial Z}\right)+4 \operatorname{tr}\left({ }^{t} V^{t}\left(Y \frac{\partial}{\partial \bar{Z}}\right) \frac{\partial}{\partial \Omega}\right) .
\end{aligned}
$$

The following differential form

$$
d v=(\operatorname{det} Y)^{-(n+m+1)}[d X] \wedge[d Y] \wedge[d U] \wedge[d V]
$$

is a $G^{J}$-invariant volume element on $\mathbb{H}_{n, m}$, where

$$
[d X]=\wedge_{\mu \leq \nu} d x_{\mu \nu}, \quad[d Y]=\wedge_{\mu \leq \nu} d y_{\mu \nu}, \quad[d U]=\wedge_{k, l} d u_{k l} \quad \text { and } \quad[d V]=\wedge_{k, l} d v_{k l} .
$$

Remark 5.2. In the special case $n=m=1$, by a direct computation, we see that the scalar curvature of $\left(\mathbb{H}_{1,1}, d s_{1,1}^{2}\right)$ is -3 . 
Remark 5.3. We let $\mathbb{M}_{1}$ and $\mathbb{M}_{2}$ be the differential operators on $\mathbb{H}_{n, m}$ defined by

$$
\mathbb{M}_{1}=4 \operatorname{tr}\left(Y \frac{\partial}{\partial Z} t\left(\frac{\partial}{\partial \bar{Z}}\right)\right)
$$

and

$$
\mathbb{M}_{2}=\Delta_{n, m}-\mathbb{M}_{1}
$$

Then $\mathbb{M}_{1}$ and $\mathbb{M}_{2}$ are invariant under the action (5.15) of $G^{J}$.

We can identify an element $g=(M,(\lambda, \mu ; \kappa))$ of $G^{J}, M=\left(\begin{array}{ll}A & B \\ C & D\end{array}\right) \in S p(n, \mathbb{R})$ with the element

$$
\left(\begin{array}{cccc}
A & 0 & B & A^{t} \mu-B^{t} \lambda \\
\lambda & I_{m} & \mu & \kappa \\
C & 0 & D & C^{t} \mu-D^{t} \lambda \\
0 & 0 & 0 & I_{m}
\end{array}\right)
$$

of $S p(m+n, \mathbb{R})$.

We set

$$
T_{*}=\frac{1}{\sqrt{2}}\left(\begin{array}{cc}
I_{m+n} & I_{m+n} \\
i I_{m+n} & -i I_{m+n}
\end{array}\right) .
$$

We now consider the group $G_{*}^{J}$ defined by

$$
G_{*}^{J}:=T_{*}^{-1} G^{J} T_{*} .
$$

If $g=(M,(\lambda, \mu ; \kappa)) \in G^{J}$ with $M=\left(\begin{array}{ll}A & B \\ C & D\end{array}\right) \in S p(n, \mathbb{R})$, then $T_{*}^{-1} g T_{*}$ is given by

$$
T_{*}^{-1} g T_{*}=\left(\begin{array}{cc}
P_{*} & Q_{*} \\
\bar{Q}_{*} & \bar{P}_{*}
\end{array}\right),
$$

where

$$
\begin{array}{cc}
P_{*} & =\left(\begin{array}{cc}
P & \frac{1}{2}\left\{Q^{t}(\lambda+i \mu)-P^{t}(\lambda-i \mu)\right\} \\
\frac{1}{2}(\lambda+i \mu) & I_{m}+i \frac{\kappa}{2}
\end{array}\right), \\
Q_{*} & =\left(\begin{array}{cc}
Q & \frac{1}{2}\left\{P^{t}(\lambda-i \mu)-Q^{t}(\lambda+i \mu)\right\} \\
\frac{1}{2}(\lambda-i \mu) & -i \frac{\kappa}{2}
\end{array}\right),
\end{array}
$$

and $P, Q$ are given by the formulas

$$
P=\frac{1}{2}\{(A+D)+i(B-C)\}
$$

and

$$
Q=\frac{1}{2}\{(A-D)-i(B+C)\} .
$$

Thus we can see that $G_{*}^{J}$ is of the form

$$
G_{*}^{J}=\left\{\left(M_{*},(\xi, \bar{\xi} ; i \kappa) \mid M_{*} \in G_{*}, \xi \in \mathbb{C}^{(m, n)}, \kappa \in \mathbb{R}^{(m, m)}\right\}\right.
$$

with the multiplication (5.14). We get the canonical action of $G_{*}^{J}$ on $\mathbb{D}_{n} \times \mathbb{C}^{(m, n)}$ defined by

$$
\left(\left(\begin{array}{ll}
\frac{P}{Q} & \frac{Q}{P}
\end{array}\right),(\xi, \bar{\xi} ; i \kappa)\right) \cdot(W, \eta)=\left((P W+Q)(\bar{Q} W+\bar{P})^{-1},(\eta+\xi W+\bar{\xi})(\bar{Q} W+\bar{P})^{-1}\right)
$$


where $\left(\frac{P}{Q} \frac{Q}{P}\right) \in G_{*}, \xi \in \mathbb{C}^{(m, n)}, \kappa \in \mathbb{R}^{(m, m)}, W \in \mathbb{D}_{n}$ and $\eta \in \mathbb{C}^{(m, n)}$. For brevity, we write $\mathbb{D}_{n, m}:=\mathbb{D}_{n} \times \mathbb{C}^{(m, n)}$. We define the partial Cayley transform $\Phi_{*}: \mathbb{D}_{n, m} \longrightarrow \mathbb{H}_{n, m}$ by

$$
\Phi_{*}(W, \eta):=\left(\left(i\left(I_{n}+W\right)\left(I_{n}-W\right)^{-1}, 2 i \eta\left(I_{n}-W\right)^{-1}\right), \quad(W, \eta) \in \mathbb{D}_{n, m} .\right.
$$

Then $\Phi_{*}$ is a biholomorphic mapping of $\mathbb{D}_{n, m}$ onto $\mathbb{H}_{n, m}$ which gives a partially bounded realization of $\mathbb{H}_{n, m}$ by $\mathbb{D}_{n, m}$.

Theorem 5.4. The action (5.15) of $G^{J}$ on $\mathbb{H}_{n, m}$ is compatible with the action (5.20) of $G_{*}^{J}$ on $\mathbb{D}_{n, m}$ through the partial Cayley transform $\Phi_{*}$ defined by (5.21). In other words, if $g_{0} \in G^{J}$ and $(W, \eta) \in \mathbb{D}_{n, m}$, then

$$
g_{0} \cdot \Phi_{*}(W, \eta)=\Phi_{*}\left(g_{*} \cdot(W, \eta)\right)
$$

where $g_{*}=T_{*}^{-1} g_{0} T_{*}$. The inverse of $\Phi_{*}$ is given by

$$
\Phi_{*}^{-1}(\Omega, Z):=\left(\left(\Omega-i I_{n}\right)\left(\Omega+i I_{n}\right)^{-1}, Z\left(\Omega+i I_{n}\right)^{-1}\right) .
$$

Proof. The proof can be found in [49].

For a coordinate $(W, \eta) \in \mathbb{D}_{n} \times \mathbb{C}^{(m, n)}$ with $W=\left(w_{\mu \nu}\right) \in \mathbb{D}_{n}$ and $\eta=\left(\eta_{k l}\right) \in \mathbb{C}^{(m, n)}$, we put

$$
\begin{aligned}
d W & =\left(d w_{\mu \nu}\right), \quad d \bar{W}=\left(d \bar{w}_{\mu \nu}\right) \\
d \eta & =\left(d \eta_{k l}\right), \quad d \bar{\eta}=\left(d \bar{\eta}_{k l}\right)
\end{aligned}
$$

and

$$
\begin{gathered}
\frac{\partial}{\partial W}=\left(\frac{1+\delta_{\mu \nu}}{2} \frac{\partial}{\partial w_{\mu \nu}}\right), \quad \frac{\partial}{\partial \bar{W}}=\left(\frac{1+\delta_{\mu \nu}}{2} \frac{\partial}{\partial \bar{w}_{\mu \nu}}\right), \\
\frac{\partial}{\partial \eta}=\left(\begin{array}{ccc}
\frac{\partial}{\partial \eta_{11}} & \cdots & \frac{\partial}{\partial \eta_{m 1}} \\
\vdots & \ddots & \vdots \\
\frac{\partial}{\partial \eta_{1 n}} & \cdots & \frac{\partial}{\partial \eta_{m n}}
\end{array}\right), \quad \frac{\partial}{\partial \bar{\eta}}=\left(\begin{array}{ccc}
\frac{\partial}{\partial \bar{\eta}_{11}} & \cdots & \frac{\partial}{\partial \bar{\eta}_{m 1}} \\
\vdots & \ddots & \vdots \\
\frac{\partial}{\partial \bar{\eta}_{1 n}} & \cdots & \frac{\partial}{\partial \bar{\eta}_{m n}}
\end{array}\right) .
\end{gathered}
$$

The author obtained the following results.

Theorem 5.5. The following metric $d s^{2}$ defined by 


$$
\begin{aligned}
\frac{1}{4} d s^{2}= & \operatorname{tr}\left(\left(I_{n}-W \bar{W}\right)^{-1} d W\left(I_{n}-\bar{W} W\right)^{-1} d \bar{W}\right)+\operatorname{tr}\left(\left(I_{n}-W \bar{W}\right)^{-1 t}(d \eta) d \bar{\eta}\right) \\
+ & \operatorname{tr}\left((\eta \bar{W}-\bar{\eta})\left(I_{n}-W \bar{W}\right)^{-1} d W\left(I_{n}-\bar{W} W\right)^{-1 t}(d \bar{\eta})\right) \\
+ & \operatorname{tr}\left((\bar{\eta} W-\eta)\left(I_{n}-\bar{W} W\right)^{-1} d \bar{W}\left(I_{n}-W \bar{W}\right)^{-1 t}(d \eta)\right) \\
- & \operatorname{tr}\left(\left(I_{n}-W \bar{W}\right)^{-1}{ }^{\prime} \eta \eta\left(I_{n}-\bar{W} W\right)^{-1} \bar{W} d W\left(I_{n}-\bar{W} W\right)^{-1} d \bar{W}\right) \\
- & \operatorname{tr}\left(W\left(I_{n}-\bar{W} W\right)^{-1} t \bar{\eta} \bar{\eta}\left(I_{n}-W \bar{W}\right)^{-1} d W\left(I_{n}-\bar{W} W\right)^{-1} d \bar{W}\right) \\
+ & \operatorname{tr}\left(\left(I_{n}-W \bar{W}\right)^{-1 t} \eta \bar{\eta}\left(I_{n}-W \bar{W}\right)^{-1} d W\left(I_{n}-\bar{W} W\right)^{-1} d \bar{W}\right) \\
+ & \operatorname{tr}\left(\left(I_{n}-\bar{W}\right)^{-1} t \bar{\eta} \eta \bar{W}\left(I_{n}-W \bar{W}\right)^{-1} d W\left(I_{n}-\bar{W} W\right)^{-1} d \bar{W}\right) \\
+ & \operatorname{tr}\left(\left(I_{n}-\bar{W}\right)^{-1}\left(I_{n}-W\right)\left(I_{n}-\bar{W} W\right)^{-1} \bar{\eta} \eta\left(I_{n}-\bar{W} W\right)^{-1}\right. \\
& \left.\times\left(I_{n}-\bar{W}\right)\left(I_{n}-W\right)^{-1} d W\left(I_{n}-\bar{W} W\right)^{-1} d \bar{W}\right) \\
- & \operatorname{tr}\left(\left(I_{n}-W \bar{W}\right)^{-1}\left(I_{n}-W\right)\left(I_{n}-\bar{W}\right)^{-1} \bar{\eta} \eta\left(I_{n}-W\right)^{-1}\right. \\
& \left.\times d W\left(I_{n}-\bar{W} W\right)^{-1} d \bar{W}\right)
\end{aligned}
$$

is a Riemannian metric on $\mathbb{D}_{n} \times \mathbb{C}^{(m, n)}$ which is invariant under the action (5.20) of the Jacobi group $G_{*}^{J}$.

Proof. The main ingredients for the proof are Theorem 5.1 and the partial Cayley transform $\Phi_{*}$. We refer to [50] for a more detailed proof.

We note that if $n=m=1$, we get

$$
\begin{aligned}
\frac{1}{4} d s^{2}= & \frac{d W d \bar{W}}{\left(1-|W|^{2}\right)^{2}}+\frac{1}{\left(1-|W|^{2}\right)} d \eta d \bar{\eta} \\
& +\frac{\left(1+|W|^{2}\right)|\eta|^{2}-\bar{W} \eta^{2}-W \bar{\eta}^{2}}{\left(1-|W|^{2}\right)^{3}} d W d \bar{W} \\
& +\frac{\eta \bar{W}-\bar{\eta}}{\left(1-|W|^{2}\right)^{2}} d W d \bar{\eta}+\frac{\bar{\eta} W-\eta}{\left(1-|W|^{2}\right)^{2}} d \bar{W} d \eta
\end{aligned}
$$


Theorem 5.6. The Laplacian $\Delta$ of $\left(\mathbb{D}_{n} \times \mathbb{C}^{(m, n)}, d s^{2}\right)$ is given by

$$
\begin{aligned}
\Delta= & \operatorname{tr}\left(\left(I_{n}-W \bar{W}\right)^{t}\left(\left(I_{n}-W \bar{W}\right) \frac{\partial}{\partial \bar{W}}\right) \frac{\partial}{\partial W}\right) \\
+ & \operatorname{tr}\left(\left(I_{n}-\bar{W} W\right) \frac{\partial}{\partial \eta}^{t}\left(\frac{\partial}{\partial \bar{\eta}}\right)\right) \\
+ & \operatorname{tr}\left({ }^{t}(\eta-\bar{\eta} W)^{t}\left(\frac{\partial}{\partial \bar{\eta}}\right)\left(I_{n}-\bar{W} W\right) \frac{\partial}{\partial W}\right) \\
+ & \operatorname{tr}\left((\bar{\eta}-\eta \bar{W})^{t}\left(\left(I_{n}-W \bar{W}\right) \frac{\partial}{\partial \bar{W}}\right) \frac{\partial}{\partial \eta}\right) \\
& -\operatorname{tr}\left(\eta \bar{W}\left(I_{n}-W \bar{W}\right)^{-1}{ }^{t} \eta^{t}\left(\frac{\partial}{\partial \bar{\eta}}\right)\left(I_{n}-\bar{W} W\right) \frac{\partial}{\partial \eta}\right) \\
& -\operatorname{tr}\left(\bar{\eta} W\left(I_{n}-\bar{W} W\right)^{-1}{ }^{t}{ }^{t}\left(\frac{\partial}{\partial \bar{\eta}}\right)\left(I_{n}-\bar{W} W\right) \frac{\partial}{\partial \eta}\right) \\
+ & \operatorname{tr}\left(\bar{\eta}\left(I_{n}-W \bar{W}\right)^{-1 t} \eta^{t}\left(\frac{\partial}{\partial \bar{\eta}}\right)\left(I_{n}-\bar{W} W\right) \frac{\partial}{\partial \eta}\right) \\
+ & \operatorname{tr}\left(\eta \bar{W} W\left(I_{n}-\bar{W} W\right)^{-1}{ }^{t} \bar{\eta}\left(\frac{\partial}{\partial \bar{\eta}}\right)\left(I_{n}-\bar{W} W\right) \frac{\partial}{\partial \eta}\right)
\end{aligned}
$$

Proof. The proof can be found in [50].

We note that if $n=m=1$, we get

$$
\begin{aligned}
\Delta= & \left(1-|W|^{2}\right)^{2} \frac{\partial^{2}}{\partial W \partial \bar{W}}+\left(1-|W|^{2}\right) \frac{\partial^{2}}{\partial \eta \partial \bar{\eta}} \\
+ & \left(1-|W|^{2}\right)(\eta-\bar{\eta} W) \frac{\partial^{2}}{\partial W \partial \bar{\eta}}+\left(1-|W|^{2}\right)(\bar{\eta}-\eta \bar{W}) \frac{\partial^{2}}{\partial \bar{W} \partial \eta} \\
& -\left(\bar{W} \eta^{2}+W \bar{\eta}^{2}\right) \frac{\partial^{2}}{\partial \eta \partial \bar{\eta}}+\left(1+|W|^{2}\right)|\eta|^{2} \frac{\partial^{2}}{\partial \eta \partial \bar{\eta}} .
\end{aligned}
$$

Remark 5.7. We see from Remark 5.3 that the following differential operators

$$
\mathbb{S}_{1}:=\sigma\left(\left(I_{n}-\bar{W} W\right) \frac{\partial}{\partial \eta} t\left(\frac{\partial}{\partial \bar{\eta}}\right)\right) \quad \text { and } \quad \mathbb{S}_{2}:=\Delta-\mathbb{S}_{1}
$$

are invariant under the action (5.20) of $G_{*}^{J}$.

We now describe the algebra $\mathbb{D}\left(\mathbb{H}_{n, m}\right)$ of all differential operators on $\mathbb{H}_{n, m}$ invariant under the action (5.15) of $G^{J}$. The adjoint action of $K$ on the tangent space $\mathfrak{p}^{J}$ of $\mathbb{H}_{n} \times \mathbb{C}^{(m, n)}$ at $\left(i I_{n}, 0\right)$ induces the action of $K$ on the complex vector space $T_{n} \times \mathbb{C}^{(m, n)}$ defined by

$$
h \cdot(\omega, z)=\left(h \omega^{t} h, z^{t} h\right),
$$

where $h \in K, w \in T_{n}$ and $z \in \mathbb{C}^{(m, n)}$. Here we regard the complex vector space $T_{n} \times \mathbb{C}^{(m, n)}$ as a real vector space. The action (5.22) induces the action $\rho$ of $K$ on the polynomial algebra $\mathrm{Pol}_{m, n}=\operatorname{Pol}\left(T_{n} \times \mathbb{C}^{(m, n)}\right)$. We denote by $\mathrm{Pol}_{m, n}^{K}$ the subalgebra of $\mathrm{Pol}_{m, n}$ consisting of 
all $K$-invariants of the action $\rho$ of $K$. Then we can show that there exists a natural linear bijection

$$
\Psi_{*}: \operatorname{Pol}_{m, n}^{K} \longrightarrow \mathbb{D}\left(\mathbb{H}_{n, m}\right)
$$

of $\operatorname{Pol}_{m, n}^{K}$ onto $\mathbb{D}\left(\mathbb{H}_{n, m}\right)$. We refer to [29], p. 287. It may be shown that the algebra $\mathbb{D}\left(\mathbb{H}_{n, m}\right)$ is generated by the images under the mapping $\Psi_{*}$ of the following invariants in $\operatorname{Pol}_{m, n}^{K}$ :

(I1) $p_{j}(\omega, z)=\operatorname{tr}\left((\omega \bar{\omega})^{j}\right)$,

(I2) $\psi_{k}^{(1)}(\omega, z)=\left(z^{t} \bar{z}\right)_{k k}$,

(I3) $\psi_{k p}^{(2)}(\omega, z)=\operatorname{Re}\left(z^{t} \bar{z}\right)_{k p}$,

(I4) $\psi_{k p}^{(3)}(\omega, z)=\operatorname{Im}\left(z^{t} \bar{z}\right)_{k p}$,

(I5) $f_{k p}^{(1)}(\omega, z)=\operatorname{Re}\left(z \bar{\omega}^{t} z\right)_{k p}$,

(I6) $f_{k p}^{(2)}(z, w)=\operatorname{Im}\left(z \bar{\omega}^{t} z\right)_{k p}$,

where $1 \leq j \leq n, 1 \leq k \leq m$ and $1 \leq k \leq p \leq m$. In the case $n=m=1$, the algebra $\mathbb{D}\left(\mathbb{H}_{1,1}\right)$ can be described explicitly as follows.

Theorem 5.8. The algebra $\mathbb{D}\left(\mathbf{H}_{1,1}\right)$ is generated by the following differential operators

$$
\begin{gathered}
D=y^{2}\left(\frac{\partial^{2}}{\partial x^{2}}+\frac{\partial^{2}}{\partial y^{2}}\right)+v^{2}\left(\frac{\partial^{2}}{\partial u^{2}}+\frac{\partial^{2}}{\partial v^{2}}\right) \\
+2 y v\left(\frac{\partial^{2}}{\partial x \partial u}+\frac{\partial^{2}}{\partial y \partial v}\right), \\
\Psi=y\left(\frac{\partial^{2}}{\partial u^{2}}+\frac{\partial^{2}}{\partial v^{2}}\right), \\
D_{1}=2 y^{2} \frac{\partial^{3}}{\partial x \partial u \partial v}-y^{2} \frac{\partial}{\partial y}\left(\frac{\partial^{2}}{\partial u^{2}}-\frac{\partial^{2}}{\partial v^{2}}\right) \\
+\left(v \frac{\partial}{\partial v}+1\right) \Psi
\end{gathered}
$$

and

$$
\begin{aligned}
D_{2}=y^{2} & \frac{\partial}{\partial x}\left(\frac{\partial^{2}}{\partial v^{2}}-\frac{\partial^{2}}{\partial u^{2}}\right)-2 y^{2} \frac{\partial^{3}}{\partial y \partial u \partial v} \\
& -v \frac{\partial}{\partial u} \Psi
\end{aligned}
$$

where $\tau=x+i y$ and $z=u+i v$ with real variables $x, y, u, v$. Moreover, we have

$$
\begin{aligned}
D \Psi-\Psi D & =2 y^{2} \frac{\partial}{\partial y}\left(\frac{\partial^{2}}{\partial u^{2}}-\frac{\partial^{2}}{\partial v^{2}}\right) \\
-4 y^{2} & \frac{\partial^{3}}{\partial x \partial u \partial v}-2\left(v \frac{\partial}{\partial v} \Psi+\Psi\right) .
\end{aligned}
$$

In particular, the algebra $\mathbb{D}\left(\mathbf{H}_{1} \times \mathbb{C}\right)$ is not commutative.

Proof. The proof can be found in $[18,47]$. 


\subsection{Maass-Jacobi Forms}

In the case $n=m=1, \mathrm{R}$. Berndt [18] introduced the notion of Maass-Jacobi forms. Now we generalize this notion to the general case.

Definition 5.9. Let

$$
\Gamma_{n, m}:=S p(n, \mathbb{Z}) \ltimes H_{\mathbb{Z}}^{(n, m)}
$$

be the discrete subgroup of $G^{J}$. A smooth function $f: \mathbb{H}_{n} \times \mathbb{C}^{(m, n)} \longrightarrow \mathbb{C}$ is called a Maass-Jacobi form on $\mathbb{H}_{n} \times \mathbb{C}^{(m, n)}$ if $f$ satisfies the following conditions (MJ1)-(MJ3) :

(MJ1) $f$ is invariant under $\Gamma_{n, m}$.

(MJ2) $f$ is an eigenfunction of the Lapalce-Beltrami operator $\Delta_{n, m}$.

(MJ3) $f$ has a polynomial growth.

It is natural to propose the following problems.

Problem A : Construct Maass-Jacobi forms.

Problem B : Find all the eigenfunctions of $\Delta_{n, m}$.

We consider the simple case $n=m=1$. A metric $d s^{2}$ on $\mathbf{H}_{1} \times \mathbb{C}$ given by

$$
\begin{gathered}
d s_{1,1}^{2}=\frac{y+v^{2}}{y^{3}}\left(d x^{2}+d y^{2}\right)+\frac{1}{y}\left(d u^{2}+d v^{2}\right) \\
-\frac{2 v}{y^{2}}(d x d u+d y d v)
\end{gathered}
$$

is a $G$-invariant Kähler metric on $\mathbf{H}_{1} \times \mathbb{C}$. Its Laplacian $\Delta_{1,1}$ is given by

$$
\begin{aligned}
\Delta_{1,1}=y^{2} & \left(\frac{\partial^{2}}{\partial x^{2}}+\frac{\partial^{2}}{\partial y^{2}}\right) \\
+ & \left(y+v^{2}\right)\left(\frac{\partial^{2}}{\partial u^{2}}+\frac{\partial^{2}}{\partial v^{2}}\right) \\
& +2 y v\left(\frac{\partial^{2}}{\partial x \partial u}+\frac{\partial^{2}}{\partial y \partial v}\right) .
\end{aligned}
$$

We provide some examples of eigenfunctions of $\Delta_{1,1}$.

(1) $h(x, y)=y^{\frac{1}{2}} K_{s-\frac{1}{2}}(2 \pi|a| y) e^{2 \pi i a x}(s \in \mathbb{C}, a \neq 0)$ with eigenvalue $s(s-1)$. Here

$$
K_{s}(z):=\frac{1}{2} \int_{0}^{\infty} \exp \left\{-\frac{z}{2}\left(t+t^{-1}\right)\right\} t^{s-1} d t
$$

where $\operatorname{Re} z>0$.

(2) $y^{s}, y^{s} x, y^{s} u(s \in \mathbb{C})$ with eigenvalue $s(s-1)$.

(3) $y^{s} v, y^{s} u v, y^{s} x v$ with eigenvalue $s(s+1)$.

(4) $x, y, u, v, x v, u v$ with eigenvalue 0 .

(5) All Maass wave forms. 


\subsection{Formal Eisenstein Series}

Let

$$
\Gamma_{1,1}^{\infty}=\left\{\left(\left(\begin{array}{cc} 
\pm 1 & m \\
0 & \pm 1
\end{array}\right),(0, n, \kappa)\right) \mid m, n, \kappa \in \mathbb{Z}\right\}
$$

be the subgroup of $\Gamma_{1,1}=S L_{2}(\mathbb{Z}) \ltimes H_{\mathbb{Z}}^{(1,1)}$. For $\gamma=\left(\left(\begin{array}{ll}a & b \\ c & d\end{array}\right),(\lambda, \mu, \kappa)\right) \in \Gamma_{1,1}$, we put $\left(\tau_{\gamma}, z_{\gamma}\right)=\gamma \cdot(\tau, z)$. That is,

$$
\begin{aligned}
& \tau_{\gamma}=(a \tau+b)(c \tau+d)^{-1}, \\
& z_{\gamma}=(z+\lambda \tau+\nu)(c \tau+d)^{-1} .
\end{aligned}
$$

We note that if $\gamma \in \Gamma_{1,1}$,

$$
\operatorname{Im} \tau_{\gamma}=\operatorname{Im} \tau, \operatorname{Im} z_{\gamma}=\operatorname{Im} z
$$

if and only if $\gamma \in \Gamma_{1,1}^{\infty}$. For $s \in \mathbb{C}$, we define an Eisenstein series formally by

$$
E_{s}(\tau, z)=\sum_{\gamma \in \Gamma_{1,1}^{\infty} \backslash \Gamma_{1,1}}\left(\operatorname{Im} \tau_{\gamma}\right)^{s} \cdot \operatorname{Im} z_{\gamma}
$$

Then $E_{s}(\tau, z)$ satisfies formally

$$
E_{s}(\gamma \cdot(\tau, z))=E_{s}(\tau, z), \quad \gamma \in \Gamma_{1,1}
$$

and

$$
\Delta E_{s}(\tau, z)=s(s+1) E_{s}(\tau, z) .
$$

But the series does not converge.

\subsection{Remarks on Fourier Expansions of Maass-Jacobi Forms}

We let $f: \mathbf{H}_{1} \times \mathbb{C} \longrightarrow \mathbb{C}$ be a Maass-Jacobi form with $\Delta f=\lambda f$. Then $f$ satisfies the following invariance relations

$$
f(\tau+n, z)=f(\tau, z) \text { for all } n \in \mathbb{Z}
$$

and

$$
f\left(\tau, z+n_{1} \tau+n_{2}\right)=f(\tau, z)
$$

for all $n_{1}, n_{2} \in \mathbb{Z}$. Therefore $f$ is a smooth function on $\mathbf{H}_{1} \times \mathbb{C}$ which is periodic in $x$ and $u$ with period 1. So $f$ has the following Fourier series

$$
f(\tau, z)=\sum_{n \in \mathbb{Z}} \sum_{r \in \mathbb{Z}} c_{n, r}(y, v) e^{2 \pi i(n x+r u)} .
$$

For two fixed integers $n$ and $r$, we have to calculate the function $c_{n, r}(y, v)$. For brevity, we put $F(y, v)=c_{n, r}(y, v)$. Then $F$ satisfies the following differential equation

$$
\left[y^{2} \frac{\partial^{2}}{\partial y^{2}}+\left(y+v^{2}\right) \frac{\partial^{2}}{\partial v^{2}}+2 y v \frac{\partial^{2}}{\partial y \partial v}\right] F=\left\{(a y+b v)^{2}+b^{2} y+\lambda\right\} F .
$$

Here $a=2 \pi n$ and $b=2 \pi r$ are constant. We note that the function $u(y)=y^{\frac{1}{2}} K_{s-\frac{1}{2}}(2 \pi|n| y)$ satisfies the above differential equation with $\lambda=s(s-1)$. Here $K_{s}(z)$ is the $K$-Bessel function before.

Problem C : Find the solutions of the above differential equations (5.23) explicitly. 


\subsection{Jacobi Forms}

Let $\rho$ be an irreducible representation of $K$ on a finite dimensional vector space $V_{\rho}$ with highest weight $\ell=\left(\ell_{1}, \cdots, \ell_{n}\right)$. Then $\rho$ is extended to a rational representation of $G L(n, \mathbb{C})$ denoted also by $\rho$. Let $\mathcal{M}$ be a symmetric half-integral semi-positive definite matrix of degree $m$. The canonical automorphic factor

$$
J_{\rho, \mathcal{M}}: G^{J} \times \mathbb{H}_{n, m} \longrightarrow G L\left(V_{\rho}\right)
$$

is given by

$$
\begin{aligned}
J_{\rho, \mathcal{M}}(g,(\Omega, Z))= & e^{-2 \pi i \sigma\left(\mathcal{M}[Z+\lambda \Omega+\mu](C \Omega+D)^{-1} C\right)} \cdot e^{2 \pi i \sigma\left(\mathcal{M}\left(\lambda \Omega \lambda^{T}+2 \lambda Z^{T}+\kappa+\mu \lambda^{T}\right)\right)} \\
& \times \rho(C \Omega+D)^{-1}
\end{aligned}
$$

where $g=(M,(\lambda, \mu, \kappa)) \in G^{J}$ with $M=\left(\begin{array}{ll}A & B \\ C & D\end{array}\right) \in S p(n, \mathbb{R})$.

For a function $f \in C^{\infty}\left(\mathbb{H}_{n, m}, V_{\rho}\right)$, we define

$$
\left(\left.f\right|_{\rho, \mathcal{M}} g\right)(\Omega, Z):=J_{\rho, \mathcal{M}}(g,(\Omega, Z)) f(g \cdot(\Omega, Z)) .
$$

Let $\Gamma_{*}$ be an arithmetic subgroup of the Siegel modular group $\Gamma_{n}=S p(n, \mathbb{Z})$ and let

$$
\Gamma_{*}^{J}:=\Gamma_{*} \ltimes H_{\mathbb{Z}}^{(n, m)}
$$

be the discrete subgroup of $\Gamma_{n, m}$. A Jacobi form of index $\mathcal{M}$ with respect to $\Gamma_{*}$ is defined to be a $V_{\rho}$-holomorphic function on $\mathbb{H}_{n, m}$ satisfying the conditions (A) and (B):

(A) $\left.f\right|_{\rho, \mathcal{M}} \gamma=f$ for all $\gamma \in \Gamma_{*}^{J}$.

(B) $f$ has a Fourier expansion of the following form

$$
f(\Omega, Z)=\sum_{T \geq 0} \sum_{R \in \mathbb{Z}^{(n, m)}} c(T, R) \cdot e^{2 \pi i \sigma(T \Omega+R Z)}
$$

where $c(T, R) \neq 0$ only if $\left(\begin{array}{cc}T & \frac{1}{2} R \\ \frac{1}{2} R & \mathcal{M}\end{array}\right) \geq 0$ and $T$ runs over the set of all half-integral matrices of degree $n$. We denote by $J_{\rho, \mathcal{M}}\left(\Gamma_{*}\right)$ the space of all Jacobi forms of index $\mathcal{M}$ with respect to $\Gamma_{*}$.

Definition. A Jacobi form $f \in J_{\rho, \mathcal{M}}\left(\Gamma_{*}\right)$ is said to be a cuspidal form if $\left(\begin{array}{cc}T & \frac{1}{2} R \\ \frac{1}{2} R & \mathcal{M}\end{array}\right)>0$ for any $T, R$ with $c(T, R) \neq 0$. A Jacobi form $f \in J_{\rho, \mathcal{M}}\left(\Gamma_{*}\right)$ is said to be a singular form if it admits a Fourier expansion such that a Fourier coefficient $c(T, R)$ vanishes unless

$$
\operatorname{det}\left(\begin{array}{cc}
T & \frac{1}{2} R \\
\frac{1}{2} R & \mathcal{M}
\end{array}\right)=0
$$

Under the assumption that $\mathcal{M}$ is positive definite, the author [44] introduced the differential operator $M_{n, m, \mathcal{M}}$ characterizing singular Jacobi forms. We let

$$
\mathcal{P}_{n}=\left\{Y \in \mathbb{R}^{(n, n)} \mid Y={ }^{t} Y>0\right\}
$$


be the open convex cone of positive definite real matrices of degree $n$. We define the differential operator $M_{n, m, \mathcal{M}}$ on $\mathcal{P}_{n} \times \mathbb{R}^{(m, n)}$ by

$$
M_{n, m, \mathcal{M}}:=\operatorname{det}(Y) \cdot \operatorname{det}\left(\frac{\partial}{\partial Y}+\frac{1}{8 \pi} t\left(\frac{\partial}{\partial V}\right) \mathcal{M}^{-1}\left(\frac{\partial}{\partial V}\right)\right)
$$

where

and

$$
Y=\left(y_{\mu \nu}\right) \in \mathcal{P}_{n}, \quad V=\left(v_{k l}\right) \in \mathbb{R}^{(m, n)}, \quad \frac{\partial}{\partial Y}=\left(\frac{1+\delta_{\mu \nu}}{2} \frac{\partial}{\partial y_{\mu \nu}}\right)
$$

$$
\frac{\partial}{\partial V}=\left(\frac{\partial}{\partial v_{k l}}\right)
$$

The author [44] proved that the following conditions $(S 1)$ and $(S 2)$ are equivalent:

$(S 1) f$ is a singular Jacobi form in $J_{\rho, \mathcal{M}}\left(\Gamma_{n}\right)$.

(S2) $f$ satisfies the differential equation $M_{n, m, \mathcal{M}} f=0$.

Let $G L_{n, m}$ be the semidirect product of the general linear group $G L(n, \mathbb{R})$ and the Heisenberg group $H_{\mathbb{R}}^{(n, m)}$ equipped with the multiplication

$$
\begin{aligned}
& (A,(\lambda, \mu ; \kappa)) \cdot\left(B,\left(\lambda^{\prime}, \mu^{\prime} ; \kappa^{\prime}\right)\right) \\
= & \left(A B,\left(\lambda B+\lambda^{\prime}, \mu^{t} B^{-1}+\mu^{\prime} ; \kappa+\kappa^{\prime}+\lambda B^{t} \mu^{\prime}-\mu^{t} B^{-1 t} \lambda^{\prime}\right)\right),
\end{aligned}
$$

where $A, B \in G L(n, \mathbb{R})$ and $(\lambda, \mu ; \kappa),\left(\lambda^{\prime}, \mu^{\prime} ; \kappa^{\prime}\right) \in H_{\mathbb{R}}^{(n, m)}$. The action (5.15) of $G^{J}$ on $\mathbb{H}_{n} \times \mathbb{C}^{(m, n)}$ yields the natural action of $G L_{n, m}$ on the homogeneous space $\mathcal{P}_{n} \times \mathbb{R}^{(m, n)}$ of non-reductive type defined by

$$
(A,(\lambda, \mu ; \kappa)) \cdot(Y, V):=\left(A Y^{t} A,(V+\lambda Y+\mu)^{t} A\right),
$$

where $A \in G L(n, \mathbb{R}),(\lambda, \mu ; \kappa) \in H_{\mathbb{R}}^{(n, m)}$ and $(Y, V) \in \mathcal{P}_{n} \times \mathbb{R}^{(m, n)}$. We can show that the differential operator $M_{n, m, \mathcal{M}}$ is invariant under the action of the subgroup

$$
G L(n, \mathbb{R}) \ltimes\left\{(0, \mu ; 0) \mid \mu \in \mathbb{R}^{(m, n)}\right\}
$$

on $\mathcal{P}_{n} \times \mathbb{R}^{(m, n)}$. Selberg [40] showed that the algebra $\mathbb{D}\left(\mathcal{P}_{n}\right)$ of all differential operators on $\mathcal{P}_{n}$ invariant under the action of $G L(n, \mathbb{R})$ on $\mathcal{P}_{n}$ defined by

$$
A \cdot Y:=A Y^{t} A, \quad A \in G L(n, \mathbb{R}), Y \in \mathcal{P}_{n}
$$

is generated by the following $n$ algebraic independent commuting differential operators

$$
\mathbb{B}_{j}=\operatorname{tr}\left(\left(Y \frac{\partial}{\partial Y}\right)^{j}\right), \quad j=1,2, \cdots, n .
$$

We refer to $[34,42]$ for more details on $\mathbb{B}_{j}$. I present the following problem.

Problem D : Describe all the generators of the algebra $\mathbb{D}\left(\mathcal{P}_{n} \times \mathbb{R}^{(m, n)}\right)$ of all differential operators on $\mathcal{P}_{n} \times \mathbb{R}^{(m, n)}$ invariant under the action (5.26) of $G L_{n, m}$ on $\mathcal{P}_{n} \times \mathbb{R}^{(m, n)}$.

I want to mention that that the theory of singular Jacobi forms may be applied to the study of the geometry of the universal abelian variety as the theory of singular modular forms to the geometry of the Siegel modular variety (cf. [46]). 
We define the lifting

$$
\Phi_{\rho, \mathcal{M}}: \mathcal{F}\left(\mathbb{H}_{n, m}, V_{\rho}\right) \longrightarrow \mathcal{F}\left(G^{J}, V_{\rho}\right)
$$

by

$$
\left(\Phi_{\rho, \mathcal{M}} f\right)(g):=\left(\left.f\right|_{\rho, \mathcal{M}} g\right)\left(i I_{n}, 0\right)=J_{\rho, \mathcal{M}}\left(g,\left(i I_{n}, 0\right)\right) f\left(g \cdot\left(i I_{n}, 0\right)\right),
$$

where $\mathcal{F}\left(\mathbb{H}_{n, m}, V_{\rho}\right)\left(\right.$ resp. $\left.\mathcal{F}\left(G^{J}, V_{\rho}\right)\right)$ denotes the space consisting of $V_{\rho^{-}}$valued functions on $\mathbb{H}_{n, m}$ (resp. $\left.G^{J}\right)$. We let

$$
\mathcal{F}_{\rho, \mathcal{M}}^{\Gamma}\left(\mathbb{H}_{n, m}\right)
$$

be the space of all $V_{\rho}$-valued functions $f$ on $\mathbb{H}_{n, m}$ satisfying the transformation formula

$$
\left.f\right|_{\rho, \mathcal{M}} \gamma=f \quad \text { for all } \gamma \in \Gamma^{J} .
$$

We let

$$
\mathcal{F}_{\rho, \mathcal{M}}^{\Gamma}\left(G^{J}\right)
$$

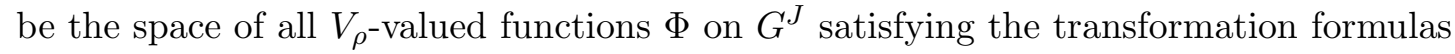

$$
\Phi(\gamma g)=\Phi(g) \text { for all } \gamma \in \Gamma^{J}, g \in G^{J}
$$

and

$$
\Phi(g \cdot(k,(0,0, \kappa)))=e^{2 \pi i \sigma(\mathcal{M} \kappa)} \rho(k)^{-1} \Phi(g)
$$

for all $(k,(0,0, \kappa)) \in K^{J}$. Then we have the isomorphism

$$
\mathcal{F}_{\rho, \mathcal{M}}^{\Gamma}\left(\mathbb{H}_{n, m}\right) \cong \mathcal{F}_{\rho, \mathcal{M}}^{\Gamma}\left(G^{J}\right)
$$

We denote by $E(\rho, \mathcal{M})$ the Hilbert space consisting of $V_{\rho^{-}}$valued measurable functions $\varphi$ on $\mathbb{H}_{n, m}$ such that

$$
\int_{\mathbb{H}_{n, m}}(\rho(Y) \varphi(\Omega, Z), \varphi(\Omega, Z)) \kappa_{\mathcal{M}}(\Omega, Z) d v<\infty
$$

where

$$
\kappa_{\mathcal{M}}(\Omega, Z)=e^{-4 \pi \sigma\left({ }^{t} V \mathcal{M} V Y^{-1}\right)} .
$$

Let $\chi$ be the unitary character of the center $\mathcal{Z}=\operatorname{Symm}\left(\mathbb{R}^{n}\right)$ defined by

$$
\chi_{\mathcal{M}}(\kappa):=e^{2 \pi i \sigma(\mathcal{M} \kappa)}, \quad \kappa \in \mathcal{Z} .
$$

The induced representation $\operatorname{Ind}_{K^{J}}^{G^{J}}\left(\rho \otimes \bar{\chi}_{\mathcal{M}}\right)$ is realized on $E(\rho, \mathcal{M})$ by

$$
\left(\operatorname{Ind}_{K^{J}}^{G^{J}}\left(\rho \otimes \bar{\chi}_{\mathcal{M}}\right)(g) \varphi\right)(\Omega, Z)=J_{\rho, \mathcal{M}}\left(g^{-1},(\Omega, Z)\right)^{-1} \varphi\left(g^{-1} \cdot(\Omega, Z)\right),
$$

where $g \in G^{J}, \varphi \in E(\rho, \mathcal{M})$ and $K^{J} \cong U(n) \times \mathcal{Z}$. The subspace

$$
H(\rho, \mathcal{M}):=\{f \in E(\rho, \mathcal{M}) \mid f \text { is holomorphic }\}
$$

is a closed $G^{J}$-invariant subspace of $E(\rho, \mathcal{M})$. Let $\pi_{\rho, \mathcal{M}}$ be the restriction of $\operatorname{Ind}_{K^{J}}^{G^{J}}\left(\rho \otimes \bar{\chi}_{\mathcal{M}}\right)$ to $H(\rho, \mathcal{M})$. We can show that if $\ell_{n}>n+\frac{1}{2}$, then $H(\rho, \mathcal{M}) \neq 0$ and $\pi_{\rho, \mathcal{M}}$ is an irreducible unitary representation of $G^{J}$ which is square integrable modulo $\mathcal{Z}$. Let

$$
L^{2}\left(\Gamma_{*}^{J} \backslash G^{J} ; V_{\rho}\right)
$$

be the Hilbert space of all $\Gamma_{*}^{J}$-invariant $V_{\rho^{-}}$-valued measurable functions on $G^{J}$ with finite norm. We denote by $L_{0}^{2}\left(\Gamma_{*}^{J} \backslash G^{J} ; V_{\rho}\right)$ the subspace consisting of functions $\varphi \in L^{2}\left(\Gamma_{*}^{J} \backslash G^{J} ; V_{\rho}\right)$ satisfying the condition

$$
\int_{\left(N^{g} \cap \Gamma_{*}^{J}\right) \backslash N^{g}} \varphi\left(n g_{0}\right) d n=0
$$


for any cuspidal subgroup $N^{g}$ of $G^{J}$ and almost all $g_{0} \in G^{J}$.

Duality Theorem. Let $\rho$ be an irreducible representation of $K$ on $V_{\rho}$ with highest weight $\ell=\left(\ell_{1}, \cdots, \ell_{n}\right) \in \mathbb{Z}^{n}$ with $\ell \geq \cdots \geq \ell_{n}$. Suppose $\ell_{n}>n+\frac{1}{2}$. Let $\mathcal{M}$ be a half-integral positive definite symmetric matrix of degree $m$. Then the multiplicity $m_{\rho, \mathcal{M}}$ of $\pi_{\rho, \mathcal{M}}$ in the regular representation $\pi_{\Gamma_{*}^{J}, \rho}$ of $G^{J}$ in $L_{0}^{2}\left(\Gamma_{*}^{J} \backslash G^{J} ; V_{\rho}\right)$ is equal to the dimension of $J_{\rho, \mathcal{M}}^{\text {cusp }}\left(\Gamma_{*}\right)$, that is,

$$
m_{\rho, \mathcal{M}}=\operatorname{dim}_{\mathbb{C}} J_{\rho, \mathcal{M}}^{\mathrm{cusp}}\left(\Gamma_{*}\right) .
$$

Remark. Berndt and Böcherer [17] proved the duality theorem for the Jacobi group in the case $n=m=1$.

\subsection{Real Analytic Eisenstein Series}

In the case $n=m=1$, T. Arakawa [1] introduced the following real analytic Eisenstein series

$$
E_{k, m, r}((\tau, z), s)\left(\tau \in \mathbb{H}_{1}, z \in \mathbb{C}\right)
$$

for each integer $r$ with $r^{2} \equiv 0(\bmod 4 m)$ and $s \in \mathbb{C}\left(m \in \mathbb{Z}^{+}, k \in \mathbb{Z}\right)$ :

$$
\begin{array}{r}
E_{k, m, r}((\tau, z), s)=\sum_{\gamma \in \Gamma_{\infty}^{J} \backslash \Gamma^{J}} e^{m}\left(\lambda^{2} \tau+2 \lambda z-\frac{c(z+\lambda \tau+\mu)^{2}}{c \tau+d}\right) \\
\times(c \tau+d)^{-k} \phi_{r, s}\left(M \circ \tau, \frac{z+\lambda \tau+\mu}{c \tau+d}\right),
\end{array}
$$

where $\Gamma^{J}=S L(2, \mathbb{Z}) \ltimes H_{\mathbb{Z}}, e^{m}(a):=e^{2 \pi i m a}$,

$$
\begin{gathered}
\gamma=\left(\left(\begin{array}{ll}
a & b \\
c & d
\end{array}\right),(\lambda, \mu, \kappa)\right) \in \Gamma^{J}, \\
\Gamma_{\infty}^{J}=\left\{\left(\left(\begin{array}{cc}
1 & n \\
0 & 1
\end{array}\right),(0, \mu, \kappa)\right) \mid n, \mu, \kappa \in \mathbb{Z}\right\}, \\
\phi_{r, s}(\tau, z):=e^{m}\left(\frac{r^{2} \tau}{4 m^{2}}+\frac{r z}{m}\right)(\operatorname{Im} \tau)^{s-\frac{1}{2}\left(k-\frac{1}{2}\right)} .
\end{gathered}
$$

It can be seen that $E_{k, m, r}((\tau, z), s)$ converges absolutely for $\operatorname{Re} s>\frac{5}{4}$ and that if $k>3$ and it is evaluated at $s=\frac{1}{2}\left(k-\frac{1}{2}\right)$, it coincides with the holomorphic Jacobi form mentioned above. Arakawa proved that under the assumption that $m$ is a square-free positive integer and $k$ is even, the above Eienstein series satisfies the functional equation

$$
E_{k, m, r}((\tau, z), 1-s)=\Phi(1-s) E_{k, m, r}((\tau, z), s)
$$

for a finite number of special integers $r$ and a certain function $\Phi(s)$ involving the Riemann zeta function and the gamma function.

\subsection{Harmonic Analysis on $\mathbb{H}_{n, m}$ and $\Gamma_{*}^{J} \backslash G^{J}$}

Since $\mathbb{H}_{n}=G / K$ is a Riemannian symmetric space of noncompact type, the Plancherel formula for $L^{2}\left(\mathbb{H}_{n}\right)$ can be obtained explicitly by the works of Helgason, van den Ban, Delorme, Schlichtkrull and others. $L^{2}\left(\mathbb{H}_{n}\right)=L^{2}(G / K)$ has no discrete series representation. 
Since the Hilbert space $L^{2}\left(\mathbb{H}_{n, m}\right)$ is isomorphic to $L^{2}\left(\mathbb{H}_{n}\right) \otimes L^{2}\left(\mathbb{R}^{(m, n)}\right)$, the Plancherel formula for $L^{2}\left(\mathbb{H}_{n, m}\right)$ can be obtained.

Let $\Gamma_{*}$ be an arithmetic subgroup of the Siegel modular group $\Gamma_{n}=S p(n, \mathbb{Z})$. From now on, for brevity, we set

$$
L^{2}:=L^{2}\left(\Gamma_{*}^{J} \backslash G^{J}\right)
$$

Then it is decomposed into

$$
L^{2}=L_{0}^{2} \oplus L_{\mathrm{res}}^{2} \oplus L_{c}^{2} .
$$

The continuous part $L_{c}^{2}$ can be understood by the theory of Eisenstein series initiated by Robert Langlands. For instance, in the case $n=m=1, L_{c}^{2}$ was described by R. Berndt $[16,18]$ in some detail.

At this moment it is hard to describe the cuspidal part $L_{0}^{2}$ in an explicit way. I propose the following problem.

Problem E : Find the trace formula for the Jacobi group $G^{J}$.

\section{REFERENCES}

[1] T. Arakawa Real analytic Eisenstein Series for the Jacobi group, Abh. Math. Sem. Univ. Hamburg 60 (1990), 131-148.

[2] J. Arthur A local trace formula, Publ. Math. I.H.E.S. No. 73 (1991).

[3] E. P. van den Ban The principal series for a reductive symmetric space, $I$. $H$-fixed distribution vectors, Ann. Scient. Éc. Norm. Sup. 21 (1988), 359-412.

[4] E. P. van den Ban The principal series for a reductive symmetric space, II. Eisenstein integrals, J. Funct. Anal. 109 (1992), 331-441.

[5] E. P. van den Ban, J. Carmona and P. Delorme Paquets d'ondes dans l'espace de Schwartz d'un espace symétrique réductif, J. Funct. Anal. 139 (1996), 225-243.

[6] E. P. van den Ban and H. Schlichtkrull Convexity for invariant differential operators on a semisimple symmetric space, Compositio Math. 89 (1993), 301-313.

[7] E. P. van den Ban and H. Schlichtkrull The most continuous part of the Plancherel decomposition for a reductive symmetric space, Ann. Math. 145 (1997), 267-364.

[8] E. P. van den Ban and H. Schlichtkrull Fourier transform on a semisimple symmetric space, Invent. Math. 145 (1997), 267-364.

[9] E. P. van den Ban and H. Schlichtkrull Fourier inversion on a reductive symmetric space, Acta Math. 182 (1999), 25-85.

[10] E. P. van den Ban and H. Schlichtkrull A residue calculus for root systems, Compositio Math. 123 (2000), 27-72.

[11] E. P. van den Ban and H. Schlichtkrull Analytic families of eigenfunctions on a reductive symmetric space, Representation Theory 5 (2001), 615-712.

[12] E. P. van den Ban and H. Schlichtkrull The Plancherel decomposition for a reductive symmetric space, I. Spherical functions. arXiv math.RT/0107063

[13] E. P. van den Ban and H. Schlichtkrull The Plancherel decomposition for a reductive symmetric space, II. Representation theory. arXiv math.RT/0111304

[14] E. P. van den Ban and H. Schlichtkrull A Paley-Wiener theorem for a reductive symmetric space. arXiv math.RT/0302232

[15] E. P. van den Ban and H. Schlichtkrull The Plancherel theorem for a reductive symmetric space, Harmonic Analysis on Symmetric Spaces-General Plancherel Theorems, edited by J.-P. Anker, B. Orsted, Progress in Math. 230, Birkhäuser, Boston (2005), 1-97.

[16] R. Berndt On the continuous part of $L^{2}\left(\Gamma^{J} \backslash G^{J}\right)$ for the Jacobi group $G^{J}$, Abh. Math. Sem. Univ. Hamburg 60 (1990), 225-248. 
[17] R. Berndt and S. Böcherer Jacobi forms and discrete series representations of the Jacobi group, Math. Z. 204 (1990), no. 1, 13-44.

[18] R. Berndt and R. Schmidt Elements of the Representation Theory of the Jacobi Group, Birkhäuser, vol. 163 (1998).

[19] J. Carmona and P. Delorme transformation de Fourier sur l'espace de Schwartz d'un espace symétriques réductif, Invent. Math. 134 (1998), 59-99.

[20] P. Delorme Troncature pour les espaces symétriques réductifs, Acta Math. 179 (1997), 41-77.

[21] P. Delorme Formule de Plancherel pour les espaces symétriques réductifs, Ann. Math. 147 (1998), 417452.

[22] P. Delorme Harmonic Analysis on Real Reductive Symmetric Spaces, Proceedings of the International Congress of Mathematicians, Vol. II, Beijing (2002), 545-554.

[23] G. van Dijk, On a class of generalized Gelfand pairs, Math. Z. 193 (1986), 581-593.

[24] M. Flensted-Jensen, Discrete series for semisimple symmetric spaces, Ann. Math. 111 (1980), $253-311$.

[25] Harish-Chandra, Discrete series for semisimple Lie groups, II. Explicit determination of the characters, Acta Math. 116 (1966), 1-111.

[26] Harish-Chandra, Harmonic analysis on real reductive Lie groups I. The theory of the constant term, J. Funct. Anal. 19 (1975), 104-204.

[27] Harish-Chandra, Harmonic analysis on real reductive Lie groups II. Wave packets in the Schwarz space, Invent. Math. 36 (1976), 1-55.

[28] Harish-Chandra, Harmonic analysis on real reductive Lie groups III. The Maass-Selberg relations and the Plancherel formula, Ann. Math. 104 (1976), 117-201.

[29] S. Helgason, Groups and geometric analysis, Academic Press (1986).

[30] A. W. Knapp, Representation Theory of Semisimple Groups, Princeton University Press, Princeton, New Jersey (1986).

[31] T. Kobayashi, Construction of discrete series for vector bundles over semisimple symmetric spaces, RIMS Kokyuroku, Kyoto University 642 (1988), 133-156.

[32] T. Kobayashi, Harmonic Analysis on Homogeneous Manifolds of Reductive Type and Unitary Representation Theory, Amer. Math. Soc. Transl. (2) Vol. 183 (1998).

[33] H. Maass, Die Differentialgleichungen in der Theorie der Siegelschen Modulfunktionen, Math. Ann. 126 (1953), 44-68.

[34] H. Maass, Siegel modular functions and Dirichlet series, Lecture Notes in Math. 216, Springer-Verlag, Berlin and New York (1971).

[35] T. Matsuki, A description of discrete series for semisimple symmetric spaces II, Advanced Studies in Pure Math. 14 (1988), 531-540.

[36] T. Matsuki and T. Oshima, A description of discrete series for semisimple symmetric spaces, Advanced Studies in Pure Math. 4 (1984), 331-390.

[37] T. Matsuki and T. Oshima, Existence conditions for discrete series for semisimple symmetric spaces, RIMS Kokyuroku, Kyoto University 642 (1988), 119-133.

[38] F. Peter and H. Weyl, Die Vollständigkeit der primitiven Darstellungen einer geschlossen kontinuierlichen Gruppe, Math. Ann. 97 (1927), 737-755.

[39] H. Schlichtkrull, Harmonic analysis on semisimple symmetric spaces, Perspective in Mathematics, Vol. 16 (1994), 91-188.

[40] A. Selberg, Harmonic analysis and discontinuous groups in weakly symmetric Riemannian spaces with applications to Dirichlet series, J. Indian Math. Soc. B. 20 (1956), 47-87.

[41] C. L. Siegel, Symplectic Geometry, Amer. J. Math. 65 (1943), 1-86; Academic Press, New York and London (1964); Gesammelte Abhandlungen, no. 41, vol. II, Springer-Verlag (1966), 274-359.

[42] A. Terras, Harmonic Analysis on Symmetric Spaces and Applications II. Springer-Verlag (1988).

[43] J.-H. Yang, Remarks on Jacobi forms of higher degree, Proceedings of the 1993 Workshop on Automorphic Forms and Related Topics (Seoul, 1993), Pyungsan Inst. Math. Sci., Seoul (1994), 33-58.

[44] J.-H. Yang, Singular Jacobi Forms, Trans. Amer. Math. Soc. 347 (6) (1995), 2041-2049.

[45] J.-H. Yang, Construction of vector valued modular forms from Jacobi forms, Canadian J. of Math. 47 (6) (1995), 1329-1339.

[46] J.-H. Yang, A Geometrical Theory of Jacobi forms of Higher Degree, Kyungpook Math. J. 40 (2) (2000), 209-237.

[47] J.-H. Yang, A note on Maass-Jacobi forms, Kyungpook Math. J. 43 (4) (2003), 547-566. 
[48] J.-H. Yang, Invariant metrics and Laplacians on the Siegel-Jacobi spaces, arXiv:math.NT/0507215 v1.

[49] J.-H. Yang, The partial Cayley transform, arXiv:math.NT/0507216 v1.

[50] J.-H. Yang, Invariant metrics and Laplacians on $\mathbb{D}_{n} \times \mathbb{C}^{(m, n)}$, arXiv:math.NT/0507217 v1.

Department of Mathematics, Inha University, Incheon 402-751, Korea

E-mail address: jhyang@inha.ac.kr 